\title{
Disinflation Shocks in the Eurozone: A DSGE Perspective
}

\author{
Patrick Fève \\ Toulouse School of Economics (University of Toulouse-GREMAQ), IDEI, \\ and Banque de France (Service de Recherche en économie et finance) \\ Julien Matheron* \\ Banque de France (Service de Recherche en économie et finance), \\ and SDFi-University of Dauphine \\ Jean-Guillaume Sahuc \\ Banque de France (Service de Recherche en économie et finance), \\ and Audencia School of Management
}

November 5, 2007

\begin{abstract}
This paper investigates the effects of disinflation policies on key macroeconomic variables. Using euro-area data and Structural VectorAutoregressions (SVARs), we first identify disinflation shocks as the only shocks that drive the inflation rate (as well as other nominal variables) to a lower level in the long-run. We find that in the immediate aftermath of a disinflation shock, the euro-area enters in a persistent recession. At the same time, the real wage and the real interest rate persistently increase. We then use the dynamic responses drawn from the SVAR model to estimate a medium-scale DSGE model. Using the estimated model, we perform counterfactual experiments. Our findings suggest that nominal wage rigidities and monetary policy gradualism have both played a prominent role in the recessionary effect of disinflation shocks in the euroarea.
\end{abstract}

Keywords: Disinflation policies, SVAR, DSGE model, Nominal rigidities, Gradualism.

JEL Class: E31, E32, E52.

\footnotetext{
*Address: Banque de France, DGEI-DIR-RECFIN, 31 rue Croix des Petits Champs, 75049, Paris Cedex 1. Email: julien.matheron@banque-france.fr. We would like to thank F. Collard, M. Dupaigne, C. Pfister, F. Portier, and J.P. Villetelle for helpful discussions. The traditional disclaimer applies. The views expressed herein are those of the authors and do not necessarily reflect those of the Banque de France.
} 


\section{Introduction}

Many economists suspect disinflation policies to be a cause of recession in industrialized countries. For example, Ball (1994) argues that most of US downturns during the seventies and the beginning of the eighties are associated with periods of monetary tightening aimed at reducing inflation. In addition, it is commonly accepted that the Volker disinflation triggered one of the most severe contraction in post World War II U.S. history (Erceg and Levin, 2003). What more, empirical studies report sizeable output losses following a disinflation policy (see Ball, 1994, Cecchetti and Rich, 2001).

The euro-area as a whole has gone through such disinflation episodes. Indeed, the annual inflation rate dramatically fell from $12 \%$ in 1980 to $4 \%$ in the late nineties. In spite of differences, inflation dynamics in individual euro-area countries all display a significant downward trend during this period. This feature reflects either a more stable monetary policy (as in Germany) or an explicit change in monetary policy (competitive disinflation in France, see Blanchard and Muet, 1993). At the same time, unemployment sharply increased and real activity was depressed, especially so during the beginning of the eighties. Obviously, these adverse effects may result from other factors and/or shocks (for example oil price shocks in the late seventies). However, shocks to monetary policy driving inflation down seem to be legitimate candidates. Indeed, as argued by Blanchard (2003), changes, either transitory or permanent, in monetary policy can have large and long-lasting effects on real activity, through very persistent changes in real factor costs.

The empirical literature based on Structural VectorAutoregressions (SVARs), usually reports a delayed and hump-shaped response of real and nominal variables to monetary policy shocks. Typically, this literature focusses on transitory monetary policy changes, representing short-run deviations from normal policy (see Christiano et al., 1999, for a thorough survey). Thus, these SVAR models are not well suited to deal with permanent changes in inflation, as observed for many industrialized countries and more specifically, for our purpose, in the euro-area during the eighties. Yet, studying disinflation-induced recessions remains a highly attractive research topic. Indeed, many researchers view the economy's dynamic responses to disinflation shocks as a natural assessment of monetary models' empirical plausibility (e.g. Furher and Moore, 1995, Coenen and Wieland, 2005). This is even truer as standard New Keynesian models are usually thought to have trouble reproducing such dynamics (Mankiw, 2001).

The aim of this paper is twofold. First, we propose a SVAR model with a minimal set of restrictions in order to assess the dynamic effect of a permanent disinflation shock in the euro-area. Second, we use the SVAR-based responses to this shock in order to estimate a medium-scale structural 
model. This estimated DSGE model is then used to perform various counterfactual experiments designed to deepen our understanding of the effects of disinflation policy shocks.

In a first step, we follow the empirical strategy proposed by Bullard and Keating (1995) and adopted by Andres et al. (2002), Dolado et al. (2000), and Vlaar (2004). This approach adapts that proposed by Blanchard and Quah (1989) in the case of permanent supply shocks. The disinflation shock is identified as the only shock that can exert a permanent effect on the long-run inflation level, as well as other nominal variables that cointegrate with inflation. In our SVAR model which includes both nominal and real variables, we obtain that the disinflation shock has long-lasting and negative effects on real activity. In particular, output persistently decreases and its dynamic response is negative during five years. Likewise, hours worked, consumption, and investment share a similar pattern, with a deeper negative response for investment. At the same time, the nominal interest rate displays such a short-run inertia that the real interest rate persistently increases. Moreover, nominal wages appear stickier in the short-run than prices, so that the real wage persistently increases too. Our results turn out to be robust to a number of perturbations to the benchmark specification. The main insight that can be drawn from this empirical analysis is that the real cost of the two factor inputs (capital and labor) seem to be associated with the disinflation-induced recession, as suggested by Blanchard (2003).

In a second step, we inspect the disinflation mechanisms through the lenses of a medium scale structural model à la Smets and Wouters (2003, 2005). Following Ireland (2007), Smets and Wouters (2005) and de Walque et al. (2007), the disinflation shock is modelled as a permanent negative shock to the central bank's inflation target. We use the estimated dynamic responses from the SVAR model as moments to be matched by the DSGE model. More precisely, relying on a limited estimation information method, ${ }^{1}$ we estimate a set of structural and policy parameters to minimize the discrepancy between responses drawn from the SVAR model and their DSGE model counterparts. Our estimation suggests that the model fits reasonably well the dynamic behavior of aggregate data after a disinflation shock. To some extent, this is a surprising result, given the alleged inability of New Keynesian models to reproduce such dynamics, according to Mankiw's (2001) criticism of this class of models.

Finally, armed with the estimated model, we perform various counterfactual experiments, in the spirit of Boivin and Giannoni (2006), Smets and Wouters (2007), Christiano et al. (2007), and

\footnotetext{
${ }^{1}$ This method has its advantages and its limits. Since it concentrates exclusively on a particular observed phenomenon, it does not specify the whole model' stochastic structure (other shocks and/or mechanisms). At the same time, the method does not pretend that the DSGE model represents the true Data Generating Process of actual data but only a useful approximation for the question under study (see Driddi, Guay and Renault, 2006).
} 
Sahuc and Smets (2007). In doing so, we resort to the estimated DSGE model as a positive assessment tool of disinflation policies. Indeed, as argued by Galí and Gertler (2007), the DSGE model can be used to run such experiments because it has explicit theoretical foundations. Our goal consists in characterizing quantitatively the propagation channels and recessionary effects of disinflation shocks. To this end, we pay particular attention to a single number called the sacrifice ratio that measures the output loss required to eliminate permanently one point of inflation. We thus make contact with a venerable literature that largely predates the current wave of DSGE models (Gordon, 1982, Gordon and King, 1982, Okun, 1978).

We investigate two main issues related to disinflation policies. The first deals with nominal rigidities and the second concerns the form of monetary policy during disinflation episodes. Both channels have been rounded as the most likely suspects explaining disinflation-induced recessions, as shown by Ball (1994). The greatest benefit of resorting to a DSGE model is our implied ability to analyze the structural mechanisms at work after a disinflation shock. Our counterfactual DSGE experiments show that the main mechanisms accounting for the level of the sacrifice ratio are: (i) nominal wage rigidities in the form of the frequency of no adjustment and the degree of indexation and (ii) the low speed of disinflation policy. These two channels contribute to persistent rises in the real wage and the real interest rate during disinflation episodes. ${ }^{2}$

The paper is organized as follows. In a second section, we present the identification of disinflation shocks and the empirical results. A third section is devoted to presenting the medium-scale structural model, the estimation results, and the counterfactual experiments obtained from the estimated DSGE model. A last section offers some concluding remarks.

\section{Identification of Disinflation Shocks}

We first describe the SVAR model and the long-run restriction used to identify the disinflation shocks. We then discuss our empirical findings with euro area data. Finally, we perform a robustness analysis on the key identification restrictions used in the SVAR model.

\footnotetext{
${ }^{2}$ As argued by Blanchard (2003), to the extent that employment and physical capital are complementary inputs, the fall in investment might translate into an even deeper fall in the labor input. We investigated this issue within the framework of our DSGE model by allowing for a less than 1 elasticity of substitution between labor and capital. For reasonable values of this elasticity, i.e. in the range $[0.5,1]$, this mechanism does not quantitatively contribute to reinforcing the recessionary effects of disinflation policies.
} 


\subsection{The SVAR Model}

In order to identify the disinflation shock, we use the following procedure. Let the vector $Z_{t}$ include the aggregate variables we are interested in. We assume that the stochastic process for $Z_{t}$ is of the form

$$
Z_{t}=A_{0}+\sum_{i=1}^{\ell} A_{i} Z_{t-i}+u_{t}
$$

where $\ell$ is the number of lags and $u_{t}$ is a zero mean disturbance with covariance matrix $\Sigma$. In the subsequent experiment, we select $\ell$ using standard information criteria.

Model (1) is estimated using euro-area quarterly data. The sample runs from 1970:1 to 2004:4. The choice of variables in $Z_{t}$ implies a trade-off. On the one hand, we would like to include as many variables as possible. However, this would imply estimating a very large number of parameters in a finite sample, thus yielding very imprecise estimates of the responses to a disinflation shock. On the other hand, a regression featuring too few variables in $Z_{t}$ could be corrupted by an omitted variable bias. We therefore choose to adopt an intermediate empirical strategy. In our benchmark experiment, $Z_{t}$ includes the following seven variables: the cyclical component of real per capita output $\left(\hat{y}_{t}\right)$, the $\log$ of the consumption-output ratio $\left(c_{t}-y_{t}\right)$, the log of the investment-output ratio $\left(x_{t}-y_{t}\right)$, the cyclical component of total hours worked per capita $\left(\hat{h}_{t}\right)$, the inflation rate $\left(\pi_{t}\right)$, the short-run nominal interest rate $\left(R_{t}\right)$, and wage inflation $\left(\pi_{t}^{w}\right)$. Except for hours worked and population, all the data are extracted from the AWM database compiled by Fagan et al. (2005). The consumption-output ratio is measured as the ratio of real private consumption expenditures (including nondurables, services, and durables expenditures) to real GDP. The investment-output ratio is defined as the ratio of real gross investment to real GDP. We measure inflation using the growth rate of the Harmonized Index of Consumer Prices (HICP). As is well known, this is the price monitored by the European Central Bank (ECB) and also the price which served to define the requirements to participate to the euro zone. Indeed, as reported in ECB (2004), "The Governing Council of the ECB has defined price stability in terms of the HICP for the euro area." In addition, this was also the price index monitored by the Deutsch Bundesbank prior to adopting the single currency. It is thus legitimate to use this price for the purpose of identifying the effects of disinflation policy shocks. Wage inflation is simply the growth rate of nominal wage compensation. ${ }^{3}$ The population series is the working age population for the euro-area, extracted from the OECD Economic Outlook. Total hours worked are extracted from the Groningen database. ${ }^{4}$ The hours

\footnotetext{
${ }^{3}$ The AWM mnemonics are as follows. Real output: YER, real consumption expenditures: PCR, real gross investment: ITR, nominal wage: WRN, short-run nominal interest rate: STN.

${ }^{4}$ These data are available at the website http://www.ggdc.net/.
} 
data are only available at annual frequency. Therefore, we resort to the Chow and Lin (1971) time disaggregation procedure to convert the series to quarterly frequency. More precisely, the growth rate of total hours is converted to quarterly frequency using the growth rate of employment as the informative covariate. The rationale for doing this is that the bulk of total hours variability is suspected to emanate essentially from changes in employment, as shown by Galí (2005) for the cases of Germany and France.

In our benchmark specification, we adopt the following definitions. The cyclical component of output, $\hat{y}_{t}$, is the residual of a regression of the log of real per capita GDP on a constant and a linear trend. The cyclical component of total hours, $\hat{h}_{t}$, is obtained as the residuals of a regression on a quadratic trend. The latter is meant to capture low frequency movements in hours worked that are attributable to institutional and/or demographic features. Our results are also robust to a specification with the raw level of hours. However, such a specification yields much wider confidence intervals.

For the purpose of identifying disinflation shocks, it is important to assess, as a preliminary step, whether inflation and other nominal variables can be characterized as integrated processes of order one over the sample period. ${ }^{5}$ To do so, we perform several Augmented Dickey Fuller (ADF) tests of unit root. More precisely, for each nominal variable, we regress the growth rate on a constant, the lagged level, and lags of the first difference. In each case, the number of lags is selected using standard information criteria. The ADF test statistic is equal to -1.52 for inflation, -2.01 for wage inflation, and -1.67 for the short-run nominal interest rate. The null hypothesis of a unit root thus cannot be rejected at the 10 percent level. Conversely, the null hypothesis of a unit root for nominal variables in first difference is rejected at conventional levels. We also investigate if there exist long-run relationships among nominal variables. To do this, we perform ADF tests on the ex-post real interest rate and real wage growth. While the difference between wage inflation and inflation unambiguously appears to be stationary (ADF test statistic of -3.91), we obtain mixed evidence when it comes to the ex-post real interest rate. An ADF test rejects its stationarity at conventional levels, but a Phillips-Perron test with a Bartlett correction is supportive of this hypothesis. Given our emphasis on a structural interpretation of disinflation shocks, we choose to impose the cointegration restrictions among nominal variables. The vector $Z_{t}$ is accordingly specified as follows:

$$
Z_{t}=\left(\Delta \pi_{t}, \hat{y}_{t}, c_{t}-y_{t}, x_{t}-y_{t}, R_{t}-\pi_{t}, \hat{h}_{t}, \pi_{t}^{w}-\pi_{t}\right)^{\prime}
$$

\footnotetext{
${ }^{5}$ We hasten to add that we view our results as a useful statistical approximation of inflation dynamics over this sample period. It is of course harder to make such an assumption for longer datasets or in the face of stable monetary policy, such as witnessed over the recent period.
} 
where $\Delta$ stands for the first-difference operator. Building on these empirical results, we follow Bullard and Keating (1995) and identify the disinflation shock as the only shock having a permanent effect on inflation (see also Andres et al., 2002, the working paper version of Coenen and Vega, 2001, Dolado et al., 2000, and Vlaar, 2004, for similar SVAR setups). This is similar in practice to the identification strategy originally proposed by Blanchard and Quah (1989) in the context of supply shocks. Formally, let us define $B(L)=\left(I_{m}-A_{1} L-\cdots-A_{\ell} L^{\ell}\right)^{-1}$, where $I_{m}$ is the identity matrix and $m$ is the number of variables in $Z_{t}$. Now, we assume that the canonical innovations are linear combinations of the structural shocks $\eta_{t}$, i.e. $u_{t}=S \eta_{t}$, for some non singular matrix S. As usual, we impose an orthogonality assumption on the structural shocks, which combined with a scale normalization implies $\mathrm{E}\left\{\eta_{t} \eta_{t}^{\prime}\right\}=I_{m}$. Since we are only identifying a single shock, we need not impose a complete set of restrictions on the matrix $S$. Let us define $C(L)=B(L) S$. Given the ordering of $Z_{t}$ in eq. (2), we simply require that $C(1)$ be lower triangular, so that only disinflation shocks can affect the long-run level of inflation. This amounts to imposing that $C(1)$ be the Cholesky factor of $B(1) \Sigma B(1)^{\prime}$. Given consistent estimates of $B(1)$ and $\Sigma$, we easily obtain an estimate for $C(1)$. Retrieving $S$ is then a simple task using the formula $S=B(1)^{-1} C(1)$.

\subsection{Results}

The lag-length $\ell=2$ in the SVAR is selected according to the Hannan-Quinn criterion (HQ). We compute confidence intervals by standard bootstrap techniques. ${ }^{6}$ To simplify the interpretation, we report the dynamic responses of consumption, investment, inflation, the short-run nominal interest rate, and wage inflation. This requires that these variables be reconstructed from the dynamic responses of $Z_{t}$. All the dynamic responses are reported in Figure 1, together with the associated confidence bands. These responses, which represent the key facts that we want to match later, are normalized so that inflation is permanently reduced by $1 \%$.

Let us first consider the responses of nominal variables. Inflation starts by sharply declining and then surges before slowly converging to its lower new long-run value. At the same time, the nominal interest rate is almost unresponsive on impact, then gradually declines, and slightly overshoots its new steady state value. This implies a significant rise in the real interest rate in the immediate aftermath of the disinflation shock. In the short-run, wage inflation decreases at a slower pace than

\footnotetext{
${ }^{6}$ We start by computing $N=1000$ bootstrap replications of the structural VAR. First, let $\left\{u_{t}\right\}_{t=1}^{T}$ denote the canonical VAR residuals. We construct $N$ new time series residuals $\left\{u_{t}(i)\right\}_{t=1}^{T}, i=1, \ldots, N$, where the $t$ th element of $\left\{u_{t}(i)\right\}_{t=1}^{T}$ is drawn with replacement from $\left\{u_{t}\right\}_{t=1}^{T}$. Using the estimated VAR coefficients and initial historical conditions, we then construct $N$ time series of $Z_{t},\left\{Z_{t}(i)\right\}_{t=1}^{T}$. For each replication, the VAR specified in eq. (1) is estimated and the impulse responses computed using the bootstrap analog of $S$.
} 
Figure 1: Impulse Response Functions
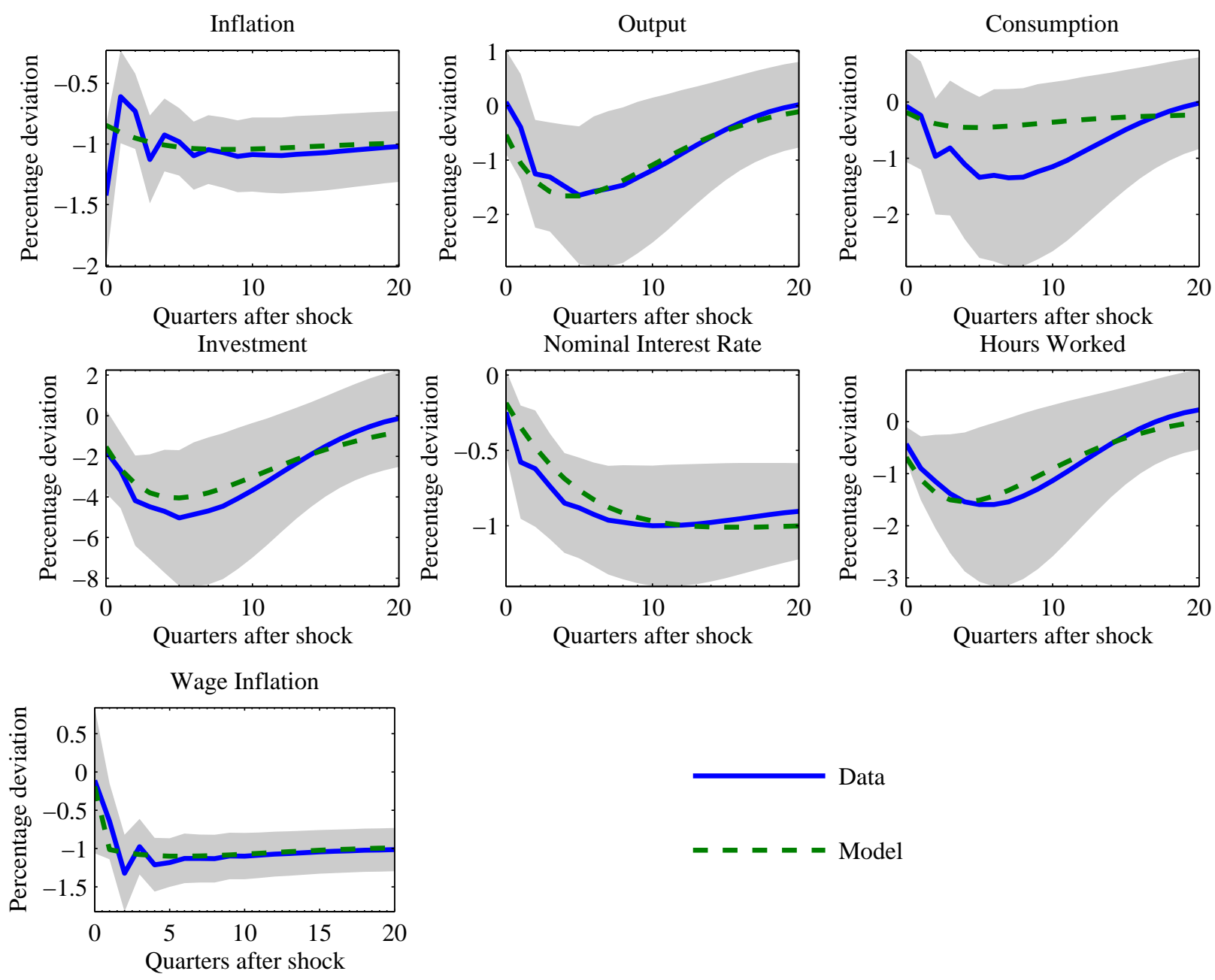

Notes: The gray area corresponds to the $90 \%$ confidence interval obtained by standard bootstrap techniques. For ease of interpretation, the size of the disinflation shock is normalized so as to generate an asymptotic inflation decrease by one percentage point.

that of inflation, implying a rise in the real wage after a disinflation shock. In the medium-run, inflation and wage inflation share very similar patterns.

When it comes to the real variables, we obtain the following results. First of all, let us consider the dynamic response of the cyclical component of output. As is clear, the disinflation shock has a long-lasting, negative, and significant effect on output. More precisely, a one percent asymptotic decrease in inflation translates into approximately a 1.5\% decline in output after about five quarters. After five years, this response is still negative. These dynamic responses imply large sacrifice ratios, defined as the cumulated responses of output divided by the annualized decrease in inflation (here, $4 \%$ ). Indeed, after five years, the sacrifice ratio is estimated to be $4.26 \%$ of cumulated forgone 
output, with a standard error of 2.37 , thus making the output loss significant at the $10 \%$ level. ${ }^{7}$ Overall, consumption declines by almost the same amount as output. This result can be explained in part by the fact that available data on consumption include durable goods expenditures. In contrast, investment experiences a drop more than twice as large as that of output. To gain some intuition, it is instructive to consider the response of the ex-ante real interest rate (not reported). The ex-ante rate is obtained as the difference between the nominal rate and the one-step ahead expected response of inflation, using the expected inflation rate implied by the SVAR model. The real rate displays a positive, persistent, and significant response to a disinflation shock. This suggests that disinflation policies have persistently increased the real cost of capital, thus leading to large declines in investment. Finally, hours worked do not react much on impact but display an inverted hump-shaped pattern in the subsequent periods. As for output, the disinflation shock has negative and significant long-lasting effects on hours worked. This dynamic response can be interpreted as the direct consequence of the persistent increase in the real wage. These patterns of dynamic responses to the disinflation shock are broadly consistent with previous results found in the literature, especially Vlaar (2004) and the working paper version of Coenen and Vega (2001). Notice, though, that we obtain deeper and more persistent recessionary effects.

Our strategy for estimating the parameters of the subsequent DSGE model focuses on only that fraction of aggregate dynamics due to disinflation shocks. As a preliminary step, it is thus important to assess how large is this fraction. To do so, we compute the contribution of the disinflation shock to the variance of the seven variables included in model (1). Table 1 reports the variance decomposition at different horizons. Concerning the nominal variables, the disinflation shock is the dominant source of their fluctuations, even in the short-run, except maybe for the nominal interest rate. For example, this shock accounts for more than $60 \%$ of inflation, more than $30 \%$ of the nominal interest rate, and more than $27 \%$ for wage inflation after four quarters. At longer horizons, the shock mechanically explains all the fluctuations in the nominal variables, by construction. Though our identification strategy imposes long-run neutrality of monetary policy shocks, the disinflation shock has a sizeable effect on real variables. It accounts for more than $10 \%$ of the variance of the cyclical component of output and hours, and $25 \%$ for investment after four quarters. This contribution is smaller for consumption (roughly 6\%). At longer horizons, say after ten years, this contribution is in between $10 \%$ and $20 \%$ for the real variables. This reflects the gradual response of the production sector to monetary policy shocks. To sum up, even though the disinflation shocks

\footnotetext{
${ }^{7}$ Our finding departs from those of Cecchetti and Rich (2001) who obtain very imprecise estimates of the sacrifice ratio in a four-variable SVAR model. Notice however that the sample data is not the same and their long-run restriction is imposed on real output. In our framework, these restrictions are imposed on nominal variables rather than on real variables.
} 
Table 1. Percentage Variance due to Disinflation Shock

\begin{tabular}{lcccc}
\hline & \multicolumn{4}{c}{ Forecast Horizon } \\
\cline { 2 - 5 } & 0 & 4 & 20 & 40 \\
\hline Inflation & 59.90 & 63.09 & 82.20 & 89.14 \\
Output & {$[7.39,81.17]$} & {$[18.75,80.73]$} & {$[60.58,88.86]$} & {$[75.81,93.57]$} \\
& 0.05 & 11.51 & 12.37 & 11.54 \\
Consumption & {$[0.02,16.59]$} & {$[1.57,34.15]$} & {$[1.60,37.28]$} & {$[1.88,36.15]$} \\
Investment & 0.06 & 6.07 & 7.87 & 7.56 \\
Nominal Interest Rate & 8.87 & 33.83 & 60.00 & 72.61 \\
& {$[0.03,19.77]$} & {$[0.83,32.61]$} & {$[0.87,37.12]$} & {$[1.28,35.48]$} \\
Hours Worked & 6.18 & 24.75 & 20.48 & 17.23 \\
& {$[0.06,26.09]$} & {$[2.93,47.32]$} & {$[2.30,47.02]$} & {$[2.53,42.93]$} \\
Wage Inflation & 16.75 & 16.80 & 11.75 & 11.34 \\
& {$[0.31,41.97]$} & {$[0.45,45.35]$} & {$[0.86,41.84]$} & {$[1.12,39.98]$} \\
& 0.12 & 27.17 & 62.64 & 75.88 \\
\hline
\end{tabular}

Notes: The figures in brackets are the $90 \%$ confidence interval, obtained by standard bootstrap techniques.

might not represent the dominant source of aggregate fluctuations at business cycle frequencies, they still account for a sizeable portion of these and thus constitute a legitimate object of study.

\subsection{Robustness Analysis}

The identification strategy adopted here calls for comments, pertaining essentially to the specification of $Z_{t}$ in eq. (2). ${ }^{8}$ Indeed, since $Z_{t}$ in eq. (2) includes other nominal variables that share the same stochastic trend as inflation, our long-run restriction also imposes that only disinflation shocks can exert a permanent effect on the nominal interest rate and wage inflation. In addition,

\footnotetext{
${ }^{8}$ Notice that the identification adopted here implies that disinflation shocks occur in each and every period. This is in contrast with the narrative approach (see Romer and Romer, 1989 and 1994) which selects a small number of episodes associated to an active disinflation policy. As noticed by Christiano, Eichenbaum and Evans (1999), an advantage of the narrative approach is that the econometrician does not have to formally specify a monetary feedback rule nor to impose a particular identification scheme to recover the responses of the economy. Such an approach is not an option for us. Indeed, analogues of the FOMC minutes are not available from either National Central Banks or the European Central Bank.
} 
by specifying the cyclical component of output and hours as deviations from deterministic trends, we implicitly impose that disinflation shocks cannot have any long-run effect whatsoever on these real variables. Moreover, by including the consumption-output ratio and the investment-output ratio in $Z_{t}$, we also impose that these variables share the same trend as output and, thus, that the disinflation shocks cannot have any long-run effect on either consumption or investment. In structural terms, the disinflation shocks can be interpreted as permanent monetary policy shocks that are neutral in the long-run. This restriction is consistent with the monetarist dictum that "inflation is always a monetary phenomenon in the long-run" (Friedman, 1968).

The restrictions documented above raise robustness problems. We examine these issues in various dimensions: omitted variables in model (1) susceptible to affect inflation dynamics (for example, the oil price), the definition of inflation (we replace the harmonized index of consumer prices by the GDP deflator), the cointegration restrictions (wage inflation and the nominal interest rate are specified in first difference), the sample period (we estimate the dynamic responses over a shorter sample), the specification of output and hours (in first difference, allowing for permanent real effect of disinflation shocks), and the monetarist dictum (allowing for an opportunistic approach to disinflation, as in Ireland, 2007). Figure 2 summarizes all our robustness experiments and compares them to our benchmark specification. This figure collects all the point estimates of the dynamic responses. Though we do not report the associated confidence interval to save space, we place much emphasis on the precision of the estimated responses. ${ }^{9}$ In all cases, the number of lags is selected according to the HQ criterion.

One may legitimately argue that the decrease in the inflation rate during the eighties can be attributable to the large decrease in oil price in the mid-eighties. To control for this decrease, we include the growth rate of the oil price as an additional variable in the SVAR model. This price is computed as the product of the West Texas Crude Oil Price and the nominal Euro- $\$$ exchange rate. Figure 2 ("Oil Price") shows that the dynamic responses are undistinguishable from those of the benchmark specification. We also investigate whether the estimated disinflation shock in our benchmark specification of the SVAR model is correlated with oil inflation and Granger caused by this variable. The contemporaneous correlation between the estimated disinflation shock and oil inflation is almost zero. Moreover, when we perform a Granger causality test, we obtain a large $P$-value for the null hypothesis on non-causality.

We now use the GDP deflator instead of the harmonized index of consumer prices in our definition of inflation in the vector $Z_{t}$. The results are left unaffected. Notice that, in comparison to the

\footnotetext{
${ }^{9} \mathrm{~A}$ technical appendix collecting and documenting this robustness analysis is available from the authors upon request.
} 
Figure 2: Dynamic Responses under Different Specifications
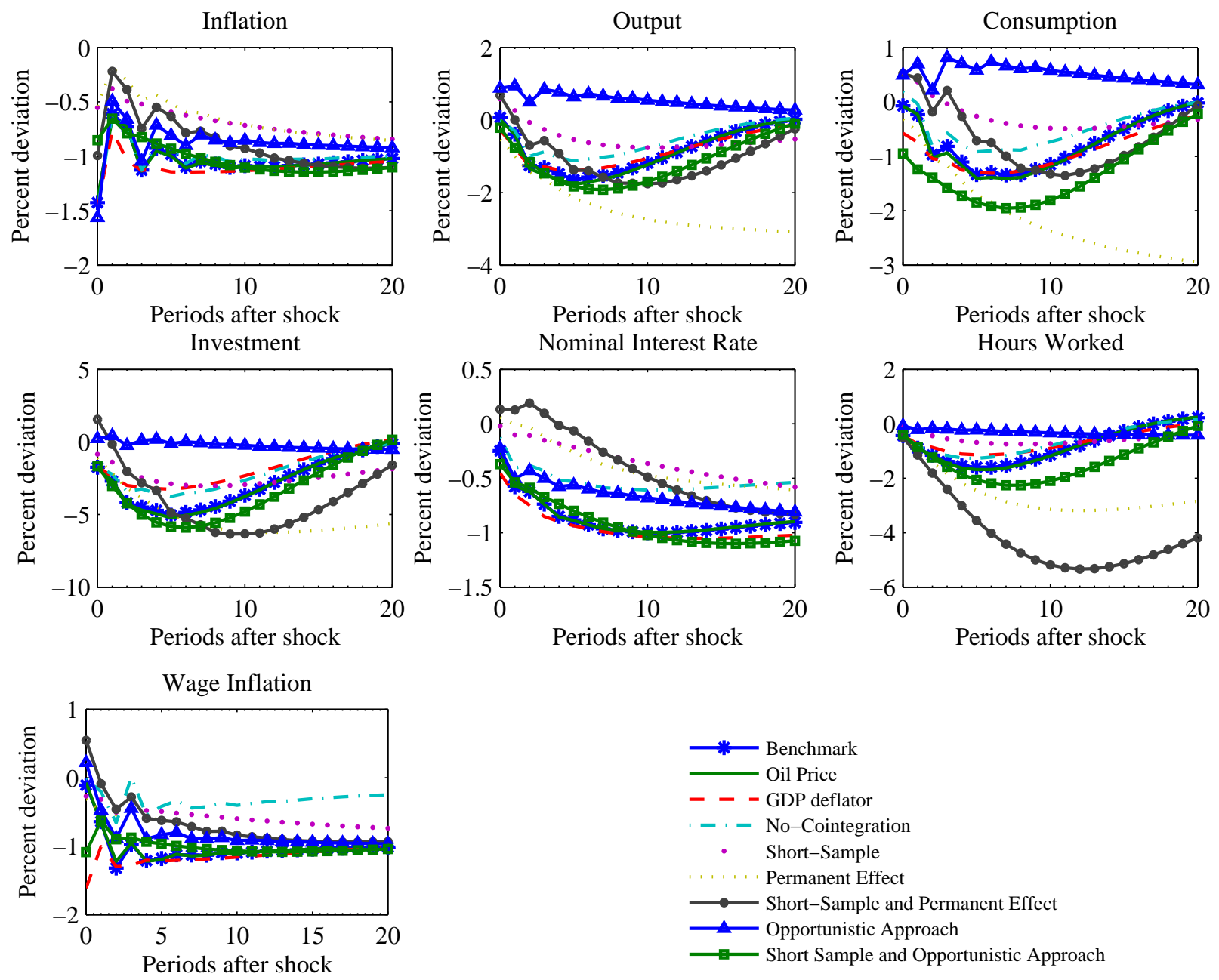

Notes: the label "Benchmark" corresponds to our preferred specification described above. The "Oil Price"label corresponds to the SVAR model with the growth rate of the oil price included in $Z_{t}$. The "GDP Deflator" label corresponds to the case where the GDP deflator is substituted for the consumer price index. The "No-Cointegration"label corresponds to the SVAR in which the cointegration restrictions are relaxed. The "Short-Sample"label reports the results obtained with the sample period 1980:1-2004:4. The "Permanent Effect"label corresponds to the case where we allow disinflation shocks to affect permanently output and hours. The "Short-Sample and Permanent Effect"label corresponds to a mix of the previous two cases. The "Opportunistic Approach"label corresponds to the case where permanent shocks to labor productivity and labor supply are allowed to affect the level of inflation. The "Short-Sample and Opportunistic Approach" corresponds to the previous case over the sample 1980:1-2004:4.

benchmark case, we obtain confidence intervals that are a little bit wider. This suggests that the GDP deflator and the HICP not only share the same long-run behavior but also share similar short-run dynamic patterns.

In the benchmark case, the SVAR model is estimated for the period 1970:1-2004:4. We investigate if the estimated dynamic responses are sensitive to the choice of sample period. We consider a shorter sample that covers the period 1980:1-2004:4. For this sample, the selected lag in the SVAR 
model is equal to one. As shown in Figure 2 ("Short-sample"), the results are almost the same but the responses are less precisely estimated (this is especially true for the estimated response of hours).

Next, we relax the restriction that nominal variables share a single stochastic trend associated to the disinflation shock. The vector $Z_{t}$ is accordingly re-specified as follows:

$$
Z_{t}=\left(\Delta \pi_{t}, \hat{y}_{t}, c_{t}-y_{t}, x_{t}-y_{t}, \Delta R_{t}, \hat{h}_{t}, \Delta \pi_{t}^{w}\right)^{\prime}
$$

We maintain the specification for the cyclical component of output and hours as deviations from deterministic trends and we use the same identification strategy for the disinflation shock as in the benchmark model. We obtain that relaxing the cointegration restrictions on nominal variables only slightly modifies our conclusions (see "No cointegration" in Figure 2). The noticeable difference does not concern the shape of the dynamics responses, but their confidence intervals. Without imposing a long-run restriction among nominal variables, the precision of the estimated dynamic responses for both nominal and real variables dramatically decreases.

In our benchmark specification, the disinflation shock cannot have a permanent effect on real variables. We relax this assumption and we now specify $Z_{t}$ as follows:

$$
Z_{t}=\left(\Delta \pi_{t}, \Delta y_{t}, c_{t}-y_{t}, x_{t}-y_{t}, R_{t}-\pi_{t}, \Delta h_{t}, \pi_{t}^{w}-\pi_{t}\right)^{\prime}
$$

When the SVAR model is estimated for the whole sample (1970:1-2004:4), the disinflation shock has a permanent detrimental effect on output, consumption, investment and hours worked (see "Permanent Effect" in Figure 2). However, the estimated responses are very sensitive to the choice of the sample period. When we estimate the model for the period 1980:1-2004:4, we find very different results (see "Short Sample and Permanent Effect" in Figure 2). For this sample period, the recessionary effect of the disinflation shocks is not permanent, in the sense that the estimated responses go back to their initial values and are not significantly different from zero. This result is in contrast with the benchmark case since the latter delivers robust estimates with respect to the sample.

Finally, we relax the monetarist dictum, thus allowing for an opportunistic approach to disinflation. Shocks that drive permanently average labor productivity (e.g. technology shocks) and hours worked (e.g. permanent labor tax shocks, permanent preference shocks on labor supply) can have a long-run effect on inflation as well as on other nominal variables. Using the same identification strategy as before, we now specify the vector $Z_{t}$ as follows:

$$
Z_{t}=\left(\Delta\left(y_{t}-h_{t}\right), \Delta h_{t}, \Delta \pi_{t}, c_{t}-y_{t}, x_{t}-y_{t}, R_{t}-\pi_{t}, \pi_{t}^{w}-\pi_{t}\right)^{\prime}
$$


With this ordering of $Z_{t}$ and the identification schemes contained in a lower triangular $C(1)$, we still maintain that the disinflation shock cannot have a long-run effect on output and hours worked (and then on consumption and investment). Notice that this specification for $Z_{t}$ is similar to that considered by Galí (1999) for the sake of identifying technology shocks. Our findings are in line with the previous experiment. When estimated on the sample 1970:1-2004:4, the estimated dynamic responses differ from those obtained in the benchmark case (see "Opportunistic Approach" in Figure 2). Now, the disinflation shock has a positive effect on output, while all nominal variables still decrease. In addition, the dynamic responses of real variables are not precisely estimated. Thus, no-clear cut evidence can be obtained from this alternative specification. Moreover, these estimated responses are not robust to the choice of sample. When the SVAR model is estimated on the shorter sample (see "Short Sample and Opportunistic Approach" in Figure 2), we obtain a persistent recessionary effect of the disinflation shock, in line with the estimations in the benchmark case. In this case, the disinflation shock is moderately contaminated by shocks that drive permanently labor productivity and hours, since the disinflation shock consistent with the monetarist dictum still represents almost $70 \%$ of the variance of inflation in the long-run.

All in all, this robustness analysis gives us confidence in the dynamic effects of disinflation previously identified. It remains to be seen whether a standard DSGE can match these moments.

\section{Structural Analysis of Disinflation Shocks}

We first briefly present the main ingredients of the DSGE model. We then discuss the estimation strategy and the empirical results. Finally, we investigate the key mechanisms at work after a disinflation shock using a counterfactual analysis.

\subsection{The Medium-Scale Structural Model}

In this section we briefly describe the structural model we will use in the subsequent quantitative analysis. ${ }^{10}$ Households maximize a separable utility function in consumption and labor effort over an infinite life horizon. Consumption appears in the utility function relative to a time-varying internal habit that depends on past consumption. Each household provides differentiated labor inputs. Monopoly power in the labor market results in an explicit wage equation and allows for

\footnotetext{
${ }^{10}$ It is a slightly modified version of the structural models presented in Smets and Wouters (2003, 2005). See also Christiano et al. (2005). See the appendix for more details about the model and the stationarity-inducing variables transformations.
} 
the introduction of sticky nominal wages as in the Calvo (1983) model (households are allowed to reset their wage each period with an exogenous probability). Households rent capital services to firms and decide how much capital to accumulate given certain costs of adjusting the capital stock. The introduction of variable capital utilization implies that as the rental price of capital changes, the capital stock can be used more or less intensively according to some cost schedule. Firms produce differentiated goods, decide on labor and capital inputs, and set prices according to the Calvo model. The Calvo model in both wage and price setting is augmented by the assumption that prices and wages that are not re-optimized in a given period are partially indexed to past inflation rates. Prices are therefore set in function of current and expected marginal costs, but are also determined by the past inflation rate. The marginal cost of production depends on the wage and the rental rate of capital. Similarly, wages also depend on past and expected future wages and inflation. Finally, the model is closed with a Taylor-type rule with partial adjustment. Here, the desired nominal interest rate is set in function of the inflation target, the inflation-gap (i.e. the difference between actual inflation and the inflation target) and the output gap.

In what follows, we briefly describe the log-linearized version of the model. Since the inflation target changes permanently, all nominal variables are deflated by this variable. Moreover, in this model, the inflation target has no long-run effect on real variables, even though it can trigger very persistent short-run dynamics for these variables.

The consumption equations:

$$
\begin{aligned}
(1-\beta b)(1-b) \hat{\lambda}_{t} & =\beta b\left(\mathrm{E}_{t}\left\{\hat{c}_{t+1}\right\}-b \hat{c}_{t}\right)-\left(\hat{c}_{t}-b \hat{c}_{t-1}\right), \\
\hat{\lambda}_{t} & =\tilde{R}_{t}+\mathrm{E}_{t}\left\{\hat{\lambda}_{t+1}-\tilde{\pi}_{t+1}-\Delta \hat{\pi}_{t+1}^{\star}\right\} .
\end{aligned}
$$

The marginal utility of wealth $\hat{\lambda}_{t}$ is a weighted average of present, past, and expected future consumption $\left(\hat{c}_{t}\right) \cdot{ }^{11}$ In turn, $\hat{\lambda}_{t}$ is linked to the ex-ante real interest rate $\tilde{R}_{t}-\mathrm{E}_{t}\left\{\tilde{\pi}_{t+1}+\Delta \hat{\pi}_{t+1}^{\star}\right\}$. Here, $\tilde{R}_{t}$ represents the nominal interest rate in deviation from the inflation target $\hat{\pi}_{t}^{\star}\left(\tilde{R}_{t} \equiv \hat{R}_{t}-\hat{\pi}_{t}^{\star}\right)$. Similarly, $\tilde{\pi}_{t}$ denotes the inflation gap $\left(\tilde{\pi}_{t} \equiv \hat{\pi}_{t}-\hat{\pi}_{t}^{\star}\right)$. The parameter $b$ captures the degree of internal habit formation in consumption and lies between zero and one. Finally $\beta$ is the subjective discount factor.

The capital accumulation equation:

$$
\hat{k}_{t+1}=(1-\delta) \hat{k}_{t}+\delta \hat{\imath}_{t}
$$

\footnotetext{
${ }^{11}$ Here and the remainder of the paper, a variable with a hat refers either to a percentage deviation from steady state or to the natural logarithm of a gross rate. With a slight abuse of notation, we also use hats to denote logarithms of non stationary variables.
} 
The capital stock $\hat{k}_{t}$ depreciates with a constant rate $\delta$. Here, $\hat{\imath}_{t}$ denotes investment.

The investment equation:

$$
\varkappa\left(\hat{\imath}_{t}-\hat{\imath}_{t-1}\right)-\beta \varkappa \mathrm{E}_{t}\left\{\hat{\imath}_{t+1}-\hat{\imath}_{t}\right\}=\hat{p}_{k, t} .
$$

Investment $\hat{\imath}_{t}$ depends on past and expected future investment and the value of the existing capital stock $\hat{p}_{k, t}$. The parameter $\varkappa$ is related to the elasticity of the investment adjustment costs.

The $Q$ equation:

$$
\mathrm{E}_{t}\left\{[1-\beta(1-\delta)] \hat{r}_{t+1}^{k}+[\beta(1-\delta)] \hat{p}_{k, t+1}+\hat{\lambda}_{t+1}\right\}=\hat{\lambda}_{t}+\hat{p}_{k, t},
$$

The value of the capital stock depends negatively on the ex-ante real interest rate $\left(\hat{\lambda}_{t}-\mathrm{E}_{t}\left\{\hat{\lambda}_{t+1}\right\}\right)$ and positively on its expected future value and on the expected real rental rate $\hat{r}_{t+1}^{k}$.

The price inflation equation:

$$
\tilde{\pi}_{t}-\gamma_{p} \tilde{\pi}_{t-1}=\frac{\left(1-\beta \alpha_{p}\right)\left(1-\alpha_{p}\right)}{\alpha_{p}} \hat{s}_{t}+\beta \mathrm{E}_{t}\left\{\left(\tilde{\pi}_{t+1}-\gamma_{p} \tilde{\pi}_{t}\right)\right\}+\gamma_{p} \zeta_{t},
$$

The inflation gap $\tilde{\pi}_{t}$ depends on its past and expected future values and on the current real marginal cost $\hat{s}_{t}$. The parameter $\alpha_{p}$ is the probability that prices cannot be reset in a given period while $\gamma_{p}$ is the degree of indexation of prices to past inflation. Finally, the residual variable $\zeta_{t}$ obeys the relation $\zeta_{t} \equiv \beta \mathrm{E}_{t}\left\{\Delta \hat{\pi}_{t+1}^{\star}\right\}-\Delta \hat{\pi}_{t}^{\star}$.

The factor prices, real marginal cost, and utilization equations

$$
\begin{aligned}
\hat{s}_{t} & =\hat{w}_{t}-\phi\left(\hat{u}_{t}+\hat{k}_{t}-\hat{h}_{t}\right), \\
\hat{s}_{t} & =\hat{r}_{t}^{k}-(\phi-1)\left(\hat{u}_{t}+\hat{k}_{t}-\hat{h}_{t}\right), \\
\hat{r}_{t}^{k} & =\sigma_{a} \hat{u}_{t} .
\end{aligned}
$$

The real wage $\hat{w}_{t}$ and the rental rate of capital $\hat{r}_{t}^{k}$ are linked to the real marginal cost according to the above equations. Here, $\hat{u}_{t}$ is the utilization rate of capital and $\hat{h}_{t}$ denotes the labor input. The parameter $\phi$ stands for the elasticity of output to the capital stock. The last equation determines the optimal degree of utilization $\hat{u}_{t}$ according to the real rental rate $\hat{r}_{t}^{k}$ and the curvature of the utilization cost function, $\sigma_{a}$.

The wage inflation equation:

$$
\tilde{\pi}_{t}^{w}-\gamma_{w} \tilde{\pi}_{t-1}=\frac{\left(1-\beta \alpha_{w}\right)\left(1-\alpha_{w}\right)}{\left(1+\omega \theta_{w}\right) \alpha_{w}}\left(\omega \hat{h}_{t}-\hat{\lambda}_{t}-\hat{w}_{t}\right)+\beta \mathrm{E}_{t}\left\{\tilde{\pi}_{t+1}^{w}-\gamma_{w} \tilde{\pi}_{t}\right\}+\gamma_{w} \zeta_{t},
$$

Nominal wage inflation (in deviation from the inflation target) $\tilde{\pi}_{t}^{w} \equiv \hat{\pi}_{t}^{w}-\hat{\pi}_{t}^{\star}$ is a function of its expected future value, past and present inflation gaps, as well as on the wage gap $\omega \hat{h}_{t}-\hat{\lambda}_{t}-\hat{w}_{t}$. 
Here the parameter $\gamma_{w}$ accounts for the degree of indexation of nominal wages to lagged inflation. The parameter $\alpha_{w}$ is the probability that nominal wages cannot be reset in a given period; $\omega$ and $\theta_{w}$ denote the inverse elasticity of labor supply and the labor demand elasticity, respectively. Finally, inflation and wage inflation are linked together according to the identity $\tilde{\pi}_{t}^{w}=\tilde{\pi}_{t}+\hat{w}_{t}-\hat{w}_{t-1}$.

The goods market equilibrium conditions:

$$
\begin{aligned}
& \hat{y}_{t}=s_{c} \hat{c}_{t}+\left(1-s_{c}\right) \hat{i}_{t}+\phi \hat{u}_{t}, \\
& \hat{y}_{t}=\mu_{p} \phi\left(\hat{u}_{t}+\hat{k}_{t}\right)+\mu_{p}(1-\phi) \hat{h}_{t} .
\end{aligned}
$$

The first equation is the resource constraint which links consumption, investment and utilization expenditures $\left(\phi \hat{u}_{t}\right)$ to aggregate output $\hat{y}_{t}$. Here $s_{c}$ is the steady state consumption-output ratio. The second equation is the production function. Because of the presence of fixed costs in production, the elasticity of aggregate production with respect to capital is $\mu_{p} \phi$ instead of $\phi$, where $\mu_{p}$ is the price markup. The fixed cost parameter is pinned down so that the share of aggregate profits in output is zero in a deterministic steady state.

The monetary policy reaction function:

$$
\hat{R}_{t}=\rho \hat{R}_{t-1}+(1-\rho)\left[\hat{\pi}_{t}^{\star}+a_{p} \tilde{\pi}_{t}+a_{y} \tilde{y}_{t}\right] .
$$

This equation is a generalized Taylor rule featuring nominal interest rate smoothing at rate $\rho$ and a variable inflation target $\hat{\pi}_{t}^{\star}$. The parameters $a_{p}$ and $a_{y}$ govern the sensitivity of the desired nominal interest rate to the inflation gap $\tilde{\pi}_{t}$ and the output gap $\tilde{y}_{t}=\hat{y}_{t}-\hat{y}_{t}^{n}$, where $\hat{y}_{t}^{n}$ represents the natural rate of output. This variable corresponds to the level of output obtained in an economy without nominal frictions, given the inherited capital stock, as in Woodford (2003). This equation ensures that (up to a constant term ignored here), any permanent change in the inflation target is reflected one to one in the nominal interest rate in the long-run. The stationary version of this equation is

$$
\tilde{R}_{t}=\rho \tilde{R}_{t-1}+(1-\rho)\left[a_{p} \tilde{\pi}_{t}+a_{y} \tilde{y}_{t}\right]-\rho \Delta \hat{\pi}_{t}^{\star}
$$

Following Ireland (2007), Smets and Wouters (2005) and de Walque, Smets and Wouters (2006), the inflation target $\hat{\pi}_{t}^{\star}$ is assumed to follow a random walk ${ }^{12}$

$$
\Delta \hat{\pi}_{t}^{\star}=\sigma_{\epsilon} \epsilon_{t}^{\star}
$$

where $\sigma_{\epsilon}>0$ and $\epsilon_{t}^{\star}$ is a zero mean and unit variance innovation to the inflation target. In what follows, we will investigate the quantitative effects of an unexpected decrease in $\epsilon_{t}^{\star}$.

\footnotetext{
${ }^{12}$ In the recent empirical literature, a similar specification has been widely adopted, see, e.g., Stock and Watson (2007) and Cogley and Sargent (2007).
} 
Obviously, as there was no single monetary policy (either in terms of adjustment speed, of inflation target or in terms of responsiveness to the economic environment) in the euro-area over the whole estimation sample adopted in our paper, the above specification warrants some words of caution. The latter is meant as a useful practical simplification of a much more complex decision making process.

\subsection{Econometric Methodology and Estimation Results}

We now turn to the estimation of the medium-scale structural model. Following Rotemberg and Woodford (1997) and Christiano et al. (2005), we adopt a Minimum-Distance Estimation technique of the structural parameters. ${ }^{13}$ The basic idea of this method is to select the value of structural and monetary policy parameters so as to minimize the discrepancy between theoretical dynamic responses and their SVAR counterparts.

More formally, let $\psi$ denote the whole set of model parameters. We partition $\psi$ in two groups, $\psi=\left(\psi_{1}^{\prime}, \psi_{2}^{\prime}\right)^{\prime}$. Let $\psi_{1}^{\prime} \equiv\left(\beta, \omega, \theta_{p}, \theta_{w}, \delta, \phi, \sigma_{\epsilon}\right)$ regroup the parameters calibrated prior to estimation. We set $\beta=0.99$, implying a steady-state annualized real interest rate of 4 percent. We impose $\omega=2$, a value similar to that obtained by Smets and Wouters (2003). The elasticity of demand for goods $\theta_{p}$ is set to 6 , while that of labor demand $\theta_{w}$ is 11 , which are quite conventional in the literature. The depreciation rate is $\delta=0.025$ and the elasticity of output with respect to capital is $\phi=0.36$. Finally, the parameter $\sigma_{\epsilon}$ associated to the disinflation shock is set to $1 \%$ as in the SVAR model. Indeed, for the selected horizon of dynamic responses drawn from the SVAR model (20 quarters), all nominal variables have approximately converged to their new long-run values.

Let $\psi_{2} \equiv\left(\gamma_{p}, \gamma_{w}, \alpha_{p}, \alpha_{w}, b, \varkappa, \sigma_{a}, \rho, a_{p}, a_{y}\right)^{\prime}$ regroup all the remaining free parameters. The latter are estimated by minimizing a measure of the distance between the empirical responses of $Z_{t}$ and their model counterparts. We define $\theta_{j}$ the vector of responses of the variables in $Z_{t}$ to a disinflation shock at horizon $j \geq 0$, as implied by the above SVAR. Then, the object which we seek to match is $\theta=\operatorname{vec}\left(\left[\theta_{0}, \theta_{1}, \ldots, \theta_{k}\right]\right)^{\prime}$ where $k$ is the selected horizon and $\operatorname{vec}(\cdot)$ is an operator that stacks the columns of a matrix. Then let $h(\cdot)$ denote the mapping from the structural parameters $\psi_{2}$ to the DSGE counterpart of $\theta$. Our estimate of $\psi_{2}$ is obtained by minimizing

$$
\left(h\left(\psi_{2}\right)-\hat{\theta}_{T}\right) V_{T}\left(h\left(\psi_{2}\right)-\hat{\theta}_{T}\right)^{\prime}
$$

where $\hat{\theta}_{T}$ is an estimate of $\theta, T$ is the sample size, and $V_{T}$ is a weighting matrix which we assume is the inverse of a matrix containing the asymptotic variances of each element of $\theta$ along its diagonal

\footnotetext{
${ }^{13}$ See also Altig et al. (2004), Amato and Laubach (2003), Boivin and Giannoni (2006) and Giannoni and Woodford (2005) among many others.
} 
Table 2. Structural Parameters

\begin{tabular}{lcl}
\hline Parameters & Value & Interpretation \\
\hline$\gamma_{p}$ & 0.000 & Price indexation parameter \\
$\gamma_{w}$ & $\begin{array}{c}(*) \\
(*)\end{array}$ & Wage indexation parameter \\
$\alpha_{p}$ & $\begin{array}{c}0.689 \\
(0.674)\end{array}$ & Proba. of no price reoptimization \\
& 0.748 & Proba. of no wage reoptimization \\
$\alpha_{w}$ & $(0.075)$ & Habit parameter \\
$b$ & 0.883 & Adjustment cost parameter \\
& $(0.090)$ & Curvature of the utilization cost function \\
$\varkappa$ & 6.892 & \\
$\sigma_{a}$ & $(5.227)$ & Interest rate smoothing \\
$\rho$ & $(0.192$ & Interest rate elasticity wrt $\hat{\pi}_{t}$ \\
$a_{p}$ & 0.775 & Interest rate elasticity wrt $\hat{y}_{t}$ \\
$a_{y}$ & $(0.108)$ & \\
\hline
\end{tabular}

Notes: The values in parentheses are the standard errors. A star refers to a parameter which hit a constraint during the course of the first stage estimation.

and zeros elsewhere. As suggested by Christiano et al. (2005), this choice of weighting matrix ensures that the model-based dynamic responses lie as much as possible inside the confidence interval of their SVAR-based counterparts.

The estimated structural parameters are reported in Table 2 and the estimated dynamic responses of the structural model in Figure 1. Three parameters hit their constraint during the course of estimation: the price indexation $\left(\gamma_{p}=0\right)$, the wage indexation $\left(\gamma_{w}=1\right)$ and the sensitivity of the desired nominal interest rate to the output gap $\left(a_{y}=0\right)$. The low price indexation is often found in the literature on euro-area data (e.g. Smets and Wouters, 2003). When it comes to the wage indexation mechanism, anticipating on our results, our finding suggests that a high value is needed to match the recessionary effects of disinflation policies. This is consistent with the view entertained by Gordon (1982), among others. Finally, setting $a_{y}=0$ is also a necessity for the purpose of matching the recessionary effects of a disinflation shock, as will appear clearer in the subsequent counterfactual analyses. This suggests that during disinflation periods, monetary policy in the euro-area as a whole was conducted without any explicit concern for real activity.

The probabilities of no price $\left(\alpha_{p}\right)$ and no wage $\left(\alpha_{w}\right)$ reoptimization are smaller than previous estimates (see Smets and Wouters, 2003, 2005). To some extent, this is not surprising since the model is asked to explain what is left of inflation's conditional persistence once inflation's stochastic 
trend has been removed. Notice that the estimation results suggest more wage stickiness than price stickiness. Recalll that, in addition, nominal wage inflation displays much more intrinsic inertia than inflation since $\gamma_{w}=1$ and $\gamma_{p}=0$. The estimated value for the habit parameter is large $(b=0.882)$ compared to previous findings with euro data. The investment adjustment cost parameter $(\varkappa=6.892)$ is not estimated precisely. The capital utilization cost parameter $\left(\sigma_{a}=0.192\right)$ is very comparable to previous estimated values. The estimated smoothing parameter in the interest rate equation is $\rho=0.775$, suggesting a substantial level of policy inertia. It is important to keep this result in mind for our counterfactual analysis. Finally, the sensitivity of the desired nominal interest rate to the inflation gap $\left(a_{p}=1.125\right)$ suggests a moderate response of monetary policy. These results are broadly consistent with the estimates obtained by Clarida et al. (1998) for the three main euro-area countries (Germany, France, Italy). For example, they report Taylor rule coefficients for inflation ranging from 0.90 to 1.31 for these three countries.

Given these estimated values, the dynamic responses of the structural model are reported in Figure 1 (dashed line) together with those from the SVAR model and with the associated confidence interval. This figure shows that the model mimics well the estimated dynamic responses from the SVAR model, with the exception of inflation in the very short-run and consumption. For inflation, this is because the model implies a persistent and smooth adjustment path, contrary to the data which display sudden changes in the very short-run. For consumption, the difference might stem from the data used in the SVAR model, since they include durable goods. For the other aggregate variables, the structural model successfully reproduces the recessionary effects of the disinflation shock, together with the short-run inertia of the nominal interest rate and the dynamic responses of wage inflation. For example, the estimated structural model implies a large sacrifice ratio for output (about 4.82) in accordance with actual data.

Thus, contrary to what one could have expected based on Mankiw's (2001) criticism of New Keynesian models, a benchmark sticky prices-sticky wages model embedding a sufficient amount of real frictions is able to mimic the aggregate dynamics triggered by a disinflation shock.

\subsection{Counter-Factual Analyses of Disinflation Policy}

Having shown that the medium-scale structural model replicates reasonably well the effects of a permanent disinflation shock on a set of real and nominal variables, we now perform counterfactual analyses. These quantitative experiments are conducted to shed light on the main mechanisms at the core of the output cost of disinflation. Here, we use the estimated model as our benchmark and we alter successively a single parameter or a set of parameters. We essentially concentrate our 
analysis on two issues that have been previously discussed in the context of disinflation policies.

The first issue concerns the role played by nominal rigidities, either on the labor or on the goods markets. Indeed, as argued by Gordon (1982), nominal wage rigidities (low frequency of adjustment and/or high degree of indexation) might be held responsible for deeper recessionary effects of disinflation. Moreover, Mankiw (1990) argues that a higher degree of price rigidities contributes to increasing the cost of disinflation. We investigate these candidate explanations by perturbing the values of the wage indexation parameter $\left(\gamma_{w}\right)$, the probability of no wage reoptimization $\left(\alpha_{w}\right)$, and the probability of no price reoptimization $\left(\alpha_{p}\right)$.

The second important issue relates to monetary policy's form, either in terms of adjustment speed or responsiveness. The literature has largely dwelt on the central and controversial question of the optimal speed of disinflation, i.e. the choice between "gradualism" and "cold turkey". On the one hand, a gradual disinflation is thought to be less costly because wages and prices display inertia and cannot adjust quickly to a permanent and sudden change in monetary policy (see Taylor, 1983). On the other hand, Sargent (1983) argues that a quick disinflation is less costly because a sharp regime change in monetary policy makes the new policy credible and thus changes downward inflation expectations. Notice also that empirical studies suggest that the speed of disinflation allows to reduce the sacrifice ratio (see Ball, 1994 and Boschen and Weise, 2001). Obviously, our DSGE model abstracts from credibility problems and is not well suited to assess Sargent's case for the euro area. At the same time, a change in the monetary policy inertia parameter $(\rho)$ in the generalized Taylor rule is a practical modelling shortcut for investigating the speed of disinflation issue. In addition, we inspect whether the responsivenesses of monetary policy to the inflation and output gaps are key ingredients of a successful disinflation policy. We thus alter the sensitivities of the desired nominal interest rate to the inflation and output gaps ( $a_{p}$ and $a_{y}$, respectively).

These various experiments are reported in figures 3 and 4 . The figures show the dynamic responses of inflation, output, consumption, investment, the nominal interest rate, hours worked, wage inflation, the real wage, and the real interest rate. In addition, for each counterfactual experiment, we compute the sacrifice ratio as a simple way to compare the cost of disinflation. Recall that this ratio is equal to 4.82 in the benchmark case. As before, we view the sacrifice ratio as a useful summary statistic of the recession deepness.

\section{Disinflation and nominal rigidities}

We first impose $\gamma_{w}=0$. In this case, the real wage does not depend on past inflation. This allows us to asses if nominal wage indexation plays an important role in the dynamics of disinflation policy. 
Figure 3: Model-Based Counterfactual Analysis: Nominal Rigidities
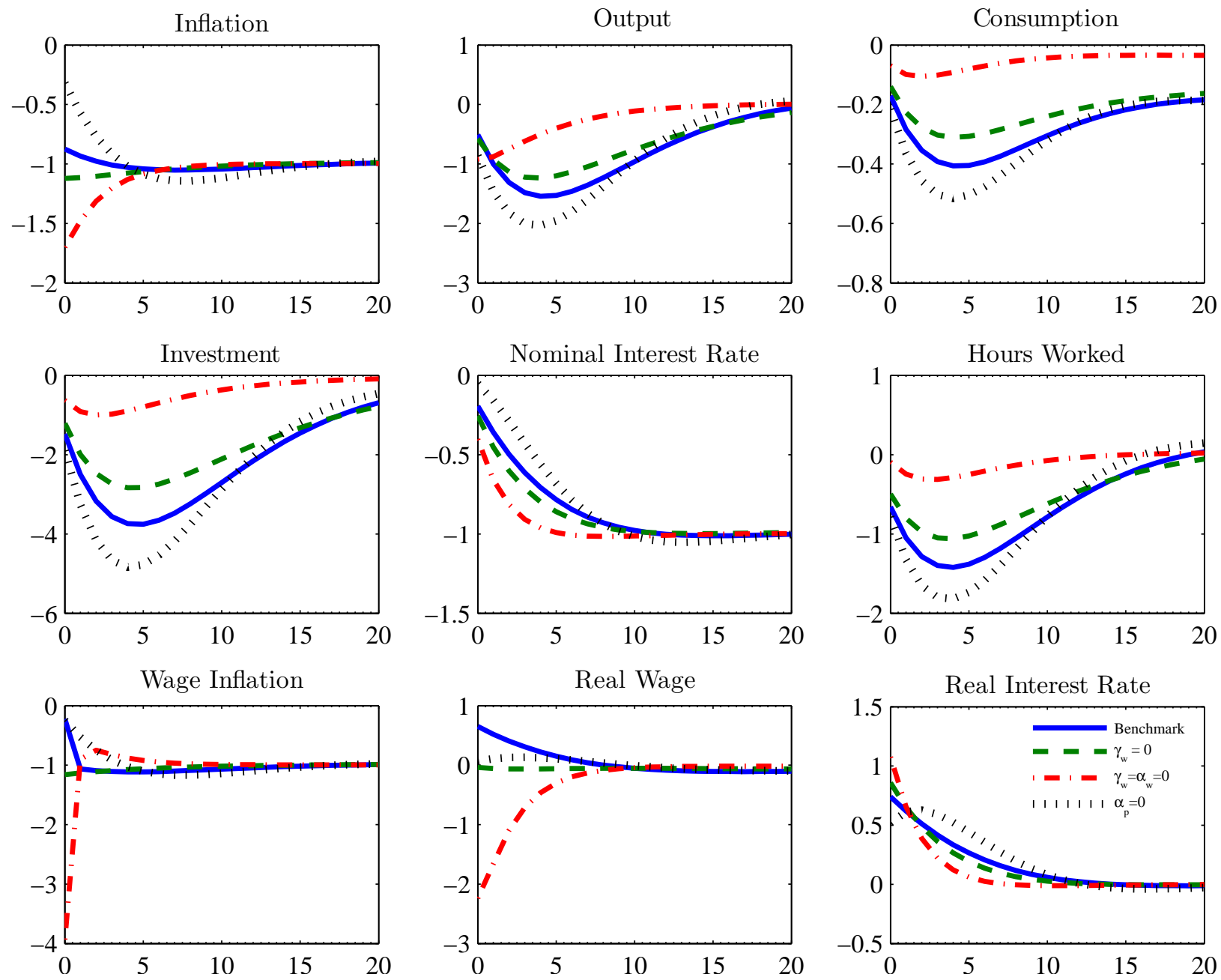

From Figure 3, we see that the long-lasting negative effect on real variables is weakened compared to the benchmark case. In this experiment, the sacrifice ratio is approximately equal to 3.70. Hence, a zero wage indexation allows to reduce the output cost of disinflation by roughly $15 \%$. Inflation and wage inflation share similar patterns, as they instantaneously drop by almost the same amount after the policy shock. It follows that the real wage is weakly reactive in the short-run. This is in contrast with the benchmark case, wherein the real wage persistently increases because of the nominal wage inertia. As a consequence, setting $\gamma_{w}=0$ implies that hours worked do not react too much. However, the negative effect on other real variables is still present, because the nominal interest rate displays higher inertia than inflation. Consequently, the real cost of capital increases after the disinflation shock and remains persistently positive. Investment then persistently decreases and thus output drops. This first experiment shows that labor market rigidities (in the form of a nominal wage indexation scheme) have potentially exacerbated the output cost of disinflation 
policy.

Second, we consider a version of the medium-scale model in which wage stickiness is eliminated while still maintaining price stickiness. The experiment is conducted by imposing $\gamma_{w}=\alpha_{w}=0$. We see in Figure 3 that a zero nominal wage rigidity dramatically reduces the output cost of disinflation. The decrease in real output is now very short-lived. Consequently, the sacrifice ratio is strongly reduced, since it is equal to 1.33 , which implies a relative reduction by $69 \%$ with respect to the benchmark case. In this model, the main mechanism at work is the large decrease in the real wage, since nominal wage quickly falls in the short-run.

Third, we impose price flexibility $\left(\alpha_{p}=0\right)$, holding nominal wage stickiness fixed. This is a simple way of assessing which of the two sources of nominal rigidity (wage or price) plays the biggest part in the disinflation story. The sacrifice ratio is now equal to 4.82 , which represents a relative increase by $12 \%$ with respect to the benchmark scenario. Indeed, in this version, the real wage displays a positive inertia in the short-run, thus implying rigid marginal cost and a fall in the labor input. This has a tendency to limit the speed at which inflation reaches its new steady state value. As a consequence, the nominal interest rate responds in a more pronounced (and hump-shaped) fashion than in the benchmark case, thus contributing to magnifying the decrease in investment, consumption, and output.

\section{Disinflation and monetary policy}

We first examine the effect of nominal interest rate inertia (see Figure 4). We set $\rho=0$, so that the desired and actual nominal interest rates exactly coincide in every period. Our quantitative finding suggests that a quick policy change results in a small recession. As mentioned before, in our model, this obtains for other reasons than credibility issues. The lesser recessionary effect stems here from the behavior of the real interest rate, as argued by Blanchard (2003). In our experiment, we find that the contractionary effect of the disinflation shock is strongly reduced under $\rho=0$. The sacrifice ratio is now equal to 1.63 , which implies a relative reduction of this cost by $62 \%$, a result quantitatively comparable to what obtains in the sticky price version of the model. This result occurs even though the real wage persistently increases and hours worked drop. The reason why is now that the real interest rate does not increase too much so that the fall in investment is overcome and a higher utilization rate of capital is substituted for the labor input.

Second, we investigate the effect of a more agressive policy with respect to the inflation gap. We set $a_{p}=3$ which represents a value twice as large as that commonly estimated for this parameter (see Smets and Wouters, 2003, 2005). This policy parameter does not significantly alter the dynamics 
Figure 4: Model-Based Counterfactual Analysis: Monetary Policy
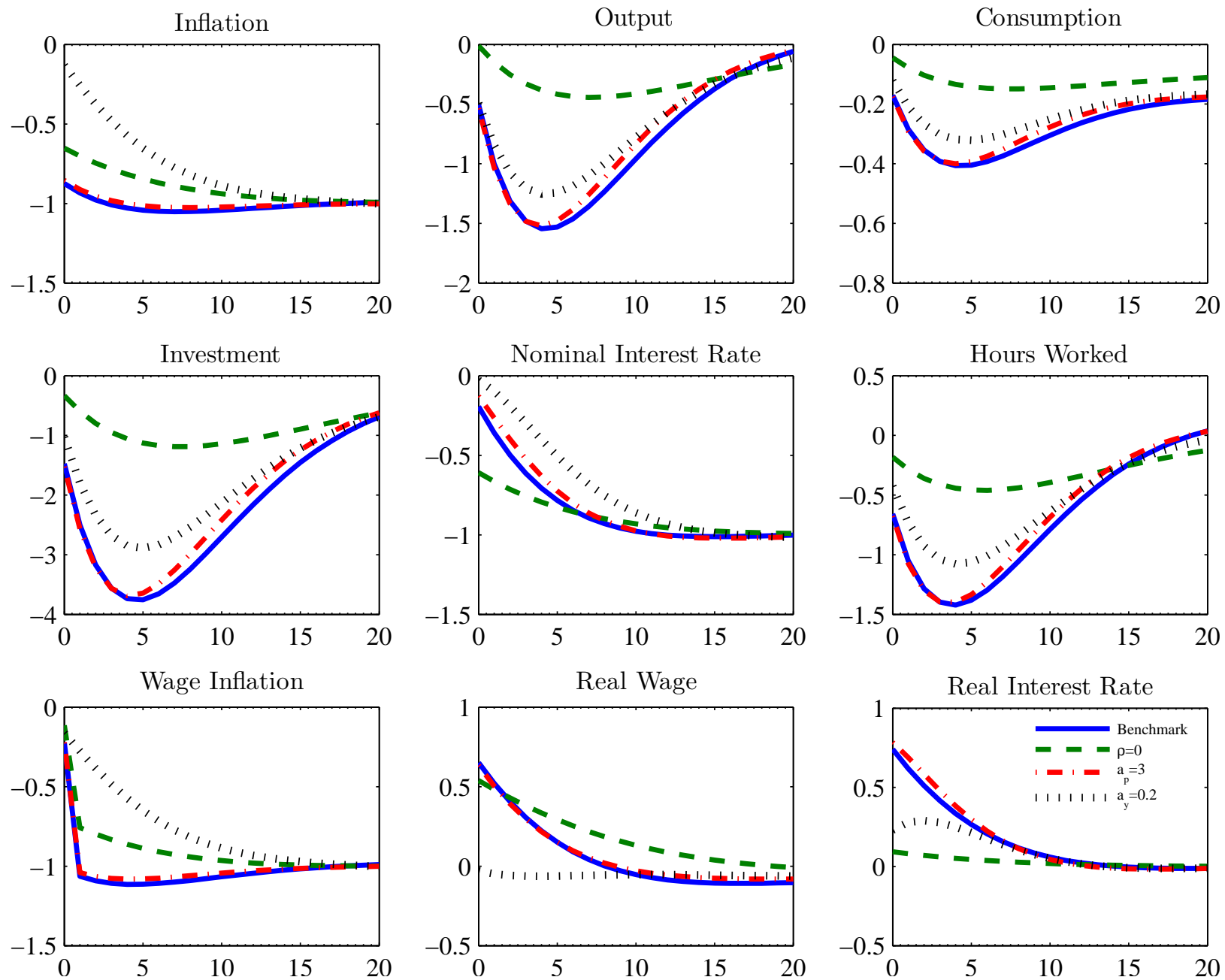

compared to what obtained in the benchmark scenario, as can be seen from Figure (4). The sacrifice ratio is now equal to 4.00 , implying a reduction by a mere $7 \%$. This reflects both the facts that aggregate inflation exhibits a low degree of inertia and that agents have already indexed their price to the new inflation target (recall that $\gamma_{p}=0$ ). Thus, given a degree of monetary policy inertia, increasing $a_{p}$ has little effect. In an economy with a higher intrisinc inflation inertia, increasing $a_{p}$ would strengthen the recessionary effects because in such a setting, the inflation gap would increase thus driving the nominal interest rate to even higher values.

Third, we consider a reactive monetary policy to the output gap. We set $a_{y}=0.2$ which represents a value twice as large as conventional values for this parameter. Contrary to the previous case, we obtain that the monetary policy sensitivity to real activity has a non zero effect on the cost of a disinflation policy. The sacrifice ratio now falls to 3.61, close to what obtains when we set $\gamma_{w}=0$. In this case, the relative output cost reduction is equal to $16 \%$. By paying attention to real 
economic activity, monetary authorities contribute to moderating the increase in the real interest rate, but only to a modest extent.

\section{Conclusion}

This paper characterizes the dynamic responses of aggregate variables to a permanent disinflation shock in the euro-area. Using a SVAR model, we obtain that this type of monetary policy has a sizeable recessionary effect on aggregate variables. Our results show that a standard medium-scale DSGE model, as currently much used in central banks, manages to match fairly well the impulse responses drawn from the SVAR. While the SVAR embeds the sufficient amount of economic theory needed to identify the disinflation shock, it is not well suited to give more than intuitions as to the economic mechanisms responsible for the overall aggregate effects of these shocks. To the contrary, the DSGE model offers a natural framework that lends itself to highlighting these mechanisms, provided that it fits the data reasonably well. Indeed, our medium-scale DSGE model turns out to capture reasonably well the aggregate dynamics triggered by a disinflation shock. Our counterfactual exercises then show that the main mechanisms at work are the following: (i) nominal wage rigidities in the form of frequency of no adjustment and the degree of indexation and (ii) the speed of disinflation policy. Interestingly, our findings echo empirical results emphasized by Ball (1994). As explained above, however, going to the hassle of specifying and estimating a DSGE model has a high payoff in terms of structural interpretation of disinflation policies. 


\section{References}

Altig, D., Christiano, L.J., Eichenbaum, M., and Linde, J. (2004) "Firm specific capital, nominal rigidities, and the business cycle", working paper \# 11034, NBER.

Amato, J. and Laubach, T. (2003) "Estimation and control of an optimization-based model with sticky prices and wages", Journal of Economic Dynamics and Control, 27, pp. 1181-1215.

Andres, J., Hernando, I., and Lóper-Salido, J. (2002) "The long-run effect of permanent disinflations", working paper \# 9825, Bank of Spain.

Ball, L. (1994) "What determines the sacrifice ratio?", in G. Mankiw (Ed.) Monetary policy, University of Chicago Press, pp. 155-88.

Blanchard, O.J. and Muet, P.A. (1993) "Competitiveness through disinflation; An assessment of French macroeconomic policy since 1983", Economic Policy, 16, pp. 12-56.

Blanchard, O.J. and Quah, D. (1989) "The dynamic effects of aggregate demand and supply disturbances", American Economic Review, 79, pp. 655-673.

Blanchard, O.J. (2003) "Monetary policy and unemployement", remarks at the Conference Monetary Policy and the Labor Market. A conference in honor of James Tobin.

Boivin, J. and Giannoni, M. (2006) "Has monetary policy become more effective?", Review of Economics and Statistics, 88, pp. 445-462.

Boschen, J. and Weise, C. (2001) "Is delayed disinflation more costly?", Southern Economic Journal, 67, pp. 701-712.

Bullard, J. and Keating, J. (1995) "The long-run relationship between inflation and output in postwar economies", Journal of Monetary Economics, 36, pp. 477-496.

Calvo, G. (1983) "Staggered prices in a utility-maximizing framework", Journal of Monetary Economics, 12 , pp. 383-398.

Cecchetti, S. and Rich, R. (2001) "Structural estimates of the US sacrifice ratio", Journal of Business and Economic Statistics, 19, pp. 416427.

Christiano, L.J., Eichenbaum, M., and Evans, C. (1999) "Monetary policy shocks: What have we learned and to what end?", in M. Woodford and J. Taylor (Eds), Handbook of Macroeconomics, North-Holland, 1999, chapter 3.

Christiano, L.J., Eichenbaum, M., and Evans, C. (2005) "Nominal rigidities and the dynamic effects of a shock to monetary policy", Journal of Political Economy, 113, pp. 1-45. 
Christiano, L.J., Motto, R., and Rostagno, M. (2007) "Shocks, structures or monetary policies? The EA and US after 2001", working paper \# 774, ECB.

Clarida, R., Galí, J., and Gertler, M. (1998) "Monetary policy rules in practice: Some international evidence", European Economic Review, 42, pp. 1033-1067

Coenen, G. and Vega, J.L. (2001) "The demand for M3 in the euro-area", Journal of Applied Econometrics, 16, pp. 727-748.

Coenen, G. and Wieland, V. (2005) "A small estimated euro-area model with rational expectations and nominal rigidities ", European Economic Review, 49, pp. 1081-1104

Cogley, T. and Sargent, T. (2007) "Inflation-gap persistence in the U.S.", mimeo.

Chow, G. and Lin, A.L. (1971) "Best linear unbiased interpolation, distribution, and extrapolation of time series by related series", Review of Economics and Statistics, 53, pp. 372-375.

de Walque, G., Smets, F., and Wouters, R. (2006) "Firm-specific production factors in a DSGE Model with Taylor price setting", International Journal of Central Banking, 2, pp. 107-154.

Dolado, J., Lóper-Salido, J., and Vega, J. (2000) "Unemployement and inflation persistence in Spain: Are there Phillips trade-offs?", Spanish Economic Review, 2, pp. 267-291.

Driddi, R., Guay, A., and Renault, E. (2007) "Indirect inference and calibration of dynamic stochastic general equilibrium model", Journal of Econometrics, 136, pp. 397-430.

Erceg, C.J. and Levin, A.T. (2003) "Imperfect credibility and inflation persistence", Journal of Monetary Economics, 50, pp. 915-944

European Central Bank (2004) The Monetary Policy of the ECB, European Central Bank.

Fagan, G., Henry, J., and Mestre, R. (2005) "An Area-Wide Model (AWM) for the euro-area", Economic Modelling, 22, pp. 39-59.

Friedman, M. (1968) "Inflation: Causes and consequences" in Dollars and Deficits: Living With Americas Economic Problems, Prentice-Hall.

Furher, J. and Moore, G. (1995) "Inflation persistence", Quarterly Journal of Economics, 110, pp. 127-159.

Galí, J. (1999) "Technology, employment, and the business cycle: Do technology schocks explain aggregate fluctuations?", American Economic Review, 89, 249-271.

Galí, J. (2005) "Trends in hours, balanced growth and the role of technology in the business cycle", Federal Reserve Bank of Saint Louis Review, 87, pp. 459-486. 
Galí, J. and Gertler, M. (2007) "Macroeconomic modeling for monetary policy evaluation", forthcoming Journal of Economic Perspective.

Giannoni, M.P. and Woodford, M. (2005) "Optimal inflation targeting rules"in Bernanke B.S. and M. Woodford (Eds.) The Inflation Targeting Debate, pp. 93-162.

Gordon, R.J. (1982) "Why stopping inflation may be costly: Evidence from fourteen historical episodes" in Hall R. (Ed.) Inflation: Causes and Effects, University of Chicago Press.

Gordon, R.J. and King, S.R. (1982) "The output cost of disinflation in traditional and Vector Autoregressive Models" Brookings Papers on Economic Activity, 1, pp. 205-244.

Ireland, P. (2007) "Changes in the Federal Reserve's inflation target: Causes and consequences", forthcoming Journal of Money, Credit and Banking.

Mankiw, N.G. (1990) "A quick refresher course in macroeconomics", Journal of Economic Literature, 28, pp. 1645-1660.

Mankiw, N.G. (2001) "The inexorable and mysterious tradeoff between inflation and unemployment", Economic Journal, 111, C45-C61.

Okun, A.M. (1978) "Efficient disinflation policies", American Economic Review, 68, pp. 348-352.

Romer, C. and Romer, D. (1989) "Does monetary policy matter? A new test in the spirit of Friedman and Schwartz", NBER Macroeconomics Annual, 4, pp. 121-170.

Romer, C. and Romer, D. (199) "Monetary policy matters", Journal of Monetary Economics, 34, pp. $75-88$.

Rotemberg, J.J. and Woodford, M. (1997) "An optimization-based econometric framework for the evaluation of monetary policy", NBER Macroeconomics Annual, pp. 297-344.

Sahuc, J.G. and Smets, F. (2007) "Differences in interest rate policy at the ECB and the Fed: An investigation with a medium-scale DSGE model", forthcoming Journal of Money, Credit, and Banking.

Sargent, T. (1983) "Stopping moderate inflations: The methods of Poincare and Thatcher", in In Inflation, Debt, and Indexation, edited by R. Dornbusch and M. Simonsen, Cambridge MA: MIT Press, pp. 54-96.

Smets, F. and Wouters, R. (2003) "An estimated dynamic stochastic general equilibrium model of the euro-area, Journal of the European Economic Association, 1, pp. 1123-1175

Smets, F. and Wouters, R. (2005) "Comparing shocks and frictions in US and euro-area business cycles: a Bayesian DSGE approach", Journal of Applied Econometrics, 20, pp. 161-183. 
Smets, F. and Wouters, R. (2007) "Shocks and frictions in US business cycles: a Bayesian DSGE approach", American Economic Review, 97, pp. 586-606.

Stock, J. and Watson, M. (2007) "Why has U.S. inflation become harder to forecast?", Journal of Money, Banking and Credit, 39, pp. 3-34.

Taylor, J.B. (1983) "Union wage settlements during a disinflation", American Economic Review, 73, pp. 980-993.

Vlaar, P. (2004) "Shocking the Eurozone", European Economic Review, 48, pp. 109-131.

Woodford, M. (2003) Interest and Prices, Princeton University Press. 


\section{Appendix}

\section{A Model Details}

This appendix details the model components and carefully derives the loglinearized price and wage equations appearing in the text.

\section{A.1 Production Side and Price Setting}

There is a unique aggregate good, $y_{t}$, which can be used either for consumption or investment purposes. The aggregate good is produced by competitive firms according to the technology

$$
y_{t}=\left(\int_{0}^{1} y_{t}(\varsigma)^{\left(\theta_{p}-1\right) / \theta_{p}} \mathrm{~d} \varsigma\right)^{\theta_{p} /\left(\theta_{p}-1\right)}
$$

where $y_{t}(\varsigma)$ is the input of intermediate good $\varsigma$, with $\varsigma \in[0,1]$. The representative aggregate good firm solves the profit maximization program

$$
\max _{\left\{y_{t}(\varsigma)\right\}} P_{t} y_{t}-\int_{0}^{1} P_{t}(\varsigma) y_{t}(\varsigma) \mathrm{d} \varsigma,
$$

where $P_{t}$ is the nominal price of the aggregate good and $P_{t}(\varsigma)$ is the nominal price of intermediate good $\varsigma$. Both are taken as given by the firm. The first order condition associated with profit maximization is

$$
y_{t}(\varsigma)=\left(\frac{P_{t}(\varsigma)}{P_{t}}\right)^{-\theta_{p}} y_{t} .
$$

Each intermediate good $\varsigma \in[0,1]$ is produced by a monopolistic firm with the corresponding index. The firm $\varsigma$ has the technology

$$
y_{t}(\varsigma)=k_{t}(\varsigma)^{\phi} n_{t}(\varsigma)^{1-\phi}-\kappa
$$

where $0<\phi<1$ is the elasticity of production with respect to capital and $\kappa$ is a fixed production cost. The terms $k_{t}(\varsigma)$ and $n_{t}(\varsigma)$, denote, respectively, the inputs of capital and aggregate labor. We assume that in each period of time, a monopolistic firm can reoptimize its price with probability $1-\alpha_{p}$, irrespective of the elapsed time since it last revised its price. If the firm cannot reoptimize its price, the latter is rescaled according to the simple revision rule

$$
P_{T}(\varsigma)=\delta_{t, T}^{p} P_{t}(\varsigma)
$$

where

$$
\delta_{t, T}^{p}=\left\{\begin{array}{ll}
\prod_{j=t}^{T-1}\left(\pi_{j+1}^{\star}\right)^{1-\gamma_{p}}\left(\pi_{j}\right)^{\gamma_{p}} & \text { if } T>t \\
1 & \text { otherwise }
\end{array},\right.
$$

where $\gamma_{p} \in(0,1)$ measures the degree of indexation to the most recently available inflation measure, $\pi_{t}$ is the (gross) inflation rate and $\pi_{t}^{\star}$ is the target (gross) inflation rate.

The real marginal cost of producing $y_{t}(\varsigma)$ units of good $\varsigma$ is formally defined as

$$
s_{t}=\left(\frac{r_{t}^{k}}{\phi}\right)^{\phi}\left(\frac{w_{t}}{1-\phi}\right)^{1-\phi}
$$

where $r_{t}^{k}$ is the real rental rate of capital and $w_{t}$ is the real wage rate. Notice that since capital and aggregate labor are supplied in economy-wide markets, the real marginal cost does not depend on $\varsigma$. 
Let $y_{t, T}^{\star}(\varsigma)$ denote the production of firm $\varsigma$ at $T$ if it last revised its price in period $t$. According to the demand function for intermediate goods, $y_{t, T}^{\star}(\varsigma)$ obeys the relationship

$$
y_{t, T}^{\star}(\varsigma)=\left(\frac{\delta_{t, T}^{p} P_{t}^{\star}(\varsigma)}{P_{T}}\right)^{-\theta_{p}} y_{T} .
$$

Thus, if drawn to reoptimize in period $t$, firm $\varsigma$ selects $P_{t}^{\star}(\varsigma)$ so as to maximize the present discounted sum of profit streams

$$
\mathrm{E}_{t} \sum_{T=t}^{\infty}\left(\beta \alpha_{p}\right)^{T-t} \frac{\lambda_{T}}{\lambda_{t}}\left[\left(\frac{\delta_{t, T}^{p} P_{t}^{\star}(\varsigma)}{P_{T}}\right)^{1-\theta_{p}} y_{T}-s_{T}\left(\frac{\delta_{t, T}^{p} P_{t}^{\star}(\varsigma)}{P_{T}}\right)^{-\theta_{p}} y_{T}\right]
$$

where $\beta^{T-t} \lambda_{T} / \lambda_{t}$ is the stochastic discount factor. The associated first order condition is

$$
\mathrm{E}_{t} \sum_{T=t}^{\infty}\left(\beta \alpha_{p}\right)^{T-t} \frac{\lambda_{T}}{\lambda_{t}} y_{t, T}^{\star}(\varsigma)\left[\frac{\delta_{t, T}^{p}}{\pi_{t, T}} p_{t}^{\star}(\varsigma)-\mu_{p}\left(\frac{y_{t, T}^{\star}(\varsigma)}{y_{T}}\right) s_{T}\right]=0
$$

where we made repeated use of eq. (7) to eliminate terms and defined

$$
p_{t}^{\star}(\varsigma) \equiv \frac{P_{t}^{\star}(\varsigma)}{P_{t}}, \quad \pi_{t, T} \equiv \frac{P_{T}}{P_{t}}, \quad \mu_{p} \equiv \frac{\theta_{p}}{\theta_{p}-1} .
$$

In steady state, we obtain the relation

$$
p^{\star}=\mu_{p} s
$$

Notice in addition that in such a symmetric steady state, all producers face the same relative price, so that $p^{\star}=1$.

Loglinearizing (8) yields

$$
\mathrm{E}_{t} \sum_{T=t}^{\infty}\left(\beta \alpha_{p}\right)^{T-t}\left\{\hat{\delta}_{t, T}^{p}-\hat{\pi}_{t, T}+\hat{p}_{t}^{\star}(\varsigma)-\hat{s}_{T}\right\}=0 .
$$

Rearranging this expression, we obtain

$$
\hat{p}_{t}^{\star}=\left(1-\beta \alpha_{p}\right) \mathrm{E}_{t} \sum_{T=t}^{\infty}\left(\beta \alpha_{p}\right)^{T-t}\left\{\left[\hat{\pi}_{t, T}-\hat{\delta}_{t, T}^{p}\right]+\hat{s}_{T}\right\} .
$$

Notice that according to this expression, the reoptimized price does not depend on $\varsigma$. We now quasi-difference this expression, which implies

$$
\hat{p}_{t}^{\star}=\left(1-\beta \alpha_{p}\right) \hat{s}_{t}+\beta \alpha_{p} \mathrm{E}_{t}\left\{\hat{p}_{t+1}^{\star}\right\}+\beta \alpha_{p}\left(1-\beta \alpha_{p}\right) \mathrm{E}_{t} \sum_{T=t+1}^{\infty}\left(\beta \alpha_{p}\right)^{T-(t+1)}\left\{\hat{\pi}_{t, T}-\hat{\delta}_{t, T}^{p}-\hat{\pi}_{t+1, T}+\hat{\delta}_{t+1, T}^{p}\right\} .
$$

In order to simplify the above expression, notice that

$$
\begin{gathered}
\hat{\pi}_{t, T}-\hat{\pi}_{t+1, T}=\hat{\pi}_{t+1}, \\
\hat{\delta}_{t+1, T}^{p}-\hat{\delta}_{t, T}^{p}=-\left[\left(1-\gamma_{p}\right) \hat{\pi}_{t+1}^{\star}+\gamma_{p} \hat{\pi}_{t}\right] .
\end{gathered}
$$

Now, let us define

$$
\tilde{\pi}_{t}=\hat{\pi}_{t}-\hat{\pi}_{t}^{\star}
$$

so that

$$
\hat{\pi}_{t, T}-\hat{\delta}_{t, T}^{p}-\hat{\pi}_{t+1, T}+\hat{\delta}_{t+1, T}^{p}=\hat{\pi}_{t+1}-\left(1-\gamma_{p}\right) \hat{\pi}_{t+1}^{\star}-\gamma_{p} \hat{\pi}_{t}=\tilde{\pi}_{t+1}-\gamma_{p} \tilde{\pi}_{t}+\gamma_{p} \Delta \hat{\pi}_{t+1}^{\star} .
$$

Using this, the above expression rewrites

$$
\hat{p}_{t}^{\star}=\left(1-\beta \alpha_{p}\right) \hat{s}_{t}+\beta \alpha_{p} \mathrm{E}_{t-1}\left\{\hat{p}_{t+1}^{\star}\right\}+\beta \alpha_{p} \mathrm{E}_{t}\left\{\tilde{\pi}_{t+1}-\gamma_{p} \tilde{\pi}_{t}+\gamma_{p} \Delta \hat{\pi}_{t+1}^{\star}\right\} .
$$


Making use of the relation defining the aggregate nominal price, we also obtain

$$
1=\left(1-\alpha_{p}\right)\left(p_{t}^{\star}\right)^{1-\theta_{p}}+\alpha_{p}\left[\frac{\left(\pi_{t}^{\star}\right)^{1-\gamma_{p}} \pi_{t-1}^{\gamma_{p}}}{\pi_{t}}\right]^{1-\theta_{p}} .
$$

Loglinearizing this expression yields

$$
\hat{p}_{t}^{\star}=\frac{\alpha_{p}}{1-\alpha_{p}}\left(\tilde{\pi}_{t}-\gamma_{p} \tilde{\pi}_{t-1}+\gamma_{p} \Delta \hat{\pi}_{t}^{\star}\right) .
$$

Plugging this relation above, we finally get

$$
\tilde{\pi}_{t}-\gamma_{p} \tilde{\pi}_{t-1}=\kappa_{p} \hat{s}_{t}+\beta \mathrm{E}_{t}\left\{\tilde{\pi}_{t+1}-\gamma_{p} \tilde{\pi}_{t}\right\}+\gamma_{p} \zeta_{t} .
$$

where

$$
\kappa_{p} \equiv \frac{\left(1-\beta \alpha_{p}\right)\left(1-\alpha_{p}\right)}{\alpha_{p}}, \quad \zeta_{t}=\beta \mathrm{E}_{t}\left\{\Delta \hat{\pi}_{t+1}^{\star}\right\}-\Delta \hat{\pi}_{t}^{\star}
$$

\section{A.2 Households and Wage Setting}

The economy is inhabited by differentiated households indexed on $[0,1]$, each of which is endowed with a specific labor type of labor. The typical household $v$ seeks to maximize

$$
\mathrm{E}_{t} \sum_{T=t}^{\infty} \beta^{T}\left\{\log \left(c_{T}-b c_{T-1}\right)-V\left(h_{T}(v)\right)\right\}
$$

subject to the constraints

$$
\begin{aligned}
& P_{T}\left[c_{T}+i_{T}+a\left(u_{T}\right) k_{T}\right]+B_{T+1} / R_{T}=W_{T}(v) h_{T}(v)+\Theta_{T}+P_{T} r_{T}^{k} u_{T} k_{T}+B_{T} \\
& k_{T+1}=(1-\delta) k_{T}+i_{T}\left(1-F\left(i_{T} / i_{T-1}\right)\right) .
\end{aligned}
$$

Here $c_{t}, i_{t}, k_{t}$ denote consumption, investment, and physical capital, respectively. The term $u_{t}$ denotes the utilization rate of physical capital and $a\left(u_{t}\right) k_{t}$ denotes the associated utilization cost, assumed to be proportional to the capital stock; $r_{t}^{k}$ is the rental rate of capital services and $W_{t}(v)$ is the nominal wage rate paid to labor of type $v$ while $h_{t}(v)$ is the quantity of type- $v$ labor supplied. $B_{t+1}$ is the quantity of nominal bonds acquired at $t$, maturing at $t+1$, and paying the gross nominal interest rate $R_{t}$. Finally $\Theta_{t}$ denotes aggregate profits redistributed by the monopolistic firms to the households. We assume that $a(u)=0$, where $u$ is the deterministic steady state value of $u_{t}$. This implies that along the balanced growth path, there are no costs associated to capital uilization. Similarly, we assume that $F(1)=F^{\prime}(1)=0$, so that adjustment costs vanish along a deterministic balanced growth path.

A typical household $v$ acts as a monopoly supplier of type- $v$ labor. Each household $v \in[0,1]$ sells its labor $h_{t}(v)$ to a competitive firm which then combines these differentiated labor inputs into an aggregate labor index $h_{t}$. This is done through the technology

$$
h_{t}=\left(\int_{0}^{1} h_{t}(v)^{\left(\theta_{w}-1\right) / \theta_{w}} \mathrm{~d} v\right)^{\theta_{w} /\left(\theta_{w}-1\right)},
$$

where $\theta_{w}>1$ is the elasticity of substitution between any two labor types. Recall that $W_{t}(v)$ denotes the nominal wage rate paid to type- $v$ labor, which labor intermediaries take as given. Let $W_{t}$ denote the nominal wage paid to aggregate labor. Accordingly, the demand curve for labor of type $v$ is

$$
h_{t}(v)=\left(\frac{W_{t}(v)}{W_{t}}\right)^{-\theta_{w}} h_{t},
$$

where the aggregate nominal wage is defined as

$$
W_{t}=\left(\int_{0}^{1} W_{t}(v)^{1-\theta_{w}} \mathrm{~d} v\right)^{1 /\left(1-\theta_{w}\right)} .
$$


Notice that equation (18) is a direct consequence of the combination of equation (17) and the zero profits condition for labor intermediaries.

It is assumed that at each point in time only a fraction $1-\alpha_{w}$ of the households can set a new wage, which will remain fixed until the next time period the household is drawn to reset its wage. The remaining households simply revise their wages according to the rule

$$
W_{T}(v)=\delta_{t, T}^{w} W_{t}(v),
$$

where

$$
\delta_{t, T}^{w}=\left\{\begin{array}{ll}
\prod_{j=t}^{T-1}\left(\pi_{j+1}^{\star}\right)^{1-\gamma_{w}}\left(\pi_{j}\right)^{\gamma_{w}} & \text { if } T>t \\
1 & \text { otherwise }
\end{array},\right.
$$

where $\gamma_{w} \in[0,1]$ measures the degree of indexation to the most recently available inflation measure, $\pi_{t}^{\star}$ is the inflation target, and $\pi_{t}$ is gross inflation.

Let us now turn our attention to the wage setting decision and define $h_{t, T}^{\star}(v)$ the supply of hours at $T$ by household $v$ if it last reoptimized its wage at $t$. In period $t$, if drawn to reoptimize, household $v$ chooses his wage rate $W_{t}^{\star}(v)$ so as to solve

$$
\max _{W_{t}^{\star}(v)} \mathrm{E}_{t} \sum_{T=t}^{\infty}\left(\beta \alpha_{w}\right)^{T-t}\left\{\lambda_{T} \frac{\delta_{t, T}^{w} W_{t}^{\star}(v)}{P_{T}} h_{t, T}^{\star}(v)-V\left(h_{t, T}^{\star}(v)\right)\right\}
$$

The associated first order condition is

$$
\mathrm{E}_{t} \sum_{T=t}^{\infty} h_{t, T}^{\star}(v)\left(\beta \alpha_{w}\right)^{T-t} h_{t, T}^{\star}(v)\left\{\lambda_{T} \frac{\delta_{t, T}^{w}}{\pi_{t, T}^{w}} \frac{w_{t}^{\star}(v)}{w_{t}} w_{T}-\frac{\theta_{w}}{\theta_{w}-1} V^{\prime}\left(h_{t, T}^{\star}(v)\right)\right\}
$$

where $w_{t}^{\star}(v) \equiv W_{t}^{\star}(v) / P_{t}$ and $\pi_{t, T}^{w} \equiv W_{T} / W_{t}$.

Loglinearizing this expression yields

$$
\mathrm{E}_{t} \sum_{t=T}^{\infty}\left(\beta \alpha_{w}\right)^{T-t}\left\{\hat{\lambda}_{T}+\hat{\delta}_{t, T}^{w}+\hat{w}_{t}^{\star}(v)+\hat{w}_{T}-\hat{w}_{t}-\hat{\pi}_{t, T}^{w}-\omega \hat{h}_{t, T}^{\star}(v)\right\}=0,
$$

with

$$
\omega=\frac{V^{\prime \prime}(h) h}{V^{\prime}(h)}
$$

The optimal labor supply is defined by

$$
h_{t, T}^{\star}(v)=\left(\frac{\delta_{t, T}^{w}}{\pi_{t, T}^{w}} \frac{w_{t}^{\star}(v)}{w_{t}}\right)^{-\theta_{w}} h_{T}
$$

which implies

$$
\hat{h}_{t, T}^{\star}(v)=\hat{h}_{T}-\theta_{w}\left(\hat{\delta}_{t, T}^{w}-\hat{\pi}_{t, T}^{w}+\hat{w}_{t}^{\star}(v)-\hat{w}_{t}\right) .
$$

Combining these loglinearized expressions yields

$$
\mathrm{E}_{t} \sum_{T=t}^{\infty}\left(\beta \alpha_{w}\right)^{T-t}\left\{\hat{\lambda}_{T}+\hat{w}_{T}-\omega \hat{h}_{T}+\left(1+\omega \theta_{w}\right)\left(\hat{\delta}_{t, T}^{w}-\hat{\pi}_{t, T}^{w}+\hat{w}_{t}^{\star}(v)-\hat{w}_{t}\right)\right\}=0 .
$$

Since all households face the same constraints, it must be the case that $W_{t}^{\star}(v)$ doesn't depend on $v$. Thus, we can drop the $v$ argument in the above equation. We finally obtain

$$
\hat{w}_{t}^{\star}-\hat{w}_{t}=\frac{1-\beta \alpha_{w}}{1+\omega \theta_{w}} \mathrm{E}_{t} \sum_{T=t}^{\infty}\left(\beta \alpha_{w}\right)^{T-t}\left\{\omega \hat{h}_{T}-\hat{\lambda}_{T}-\hat{w}_{T}+\left(1+\omega \theta_{w}\right)\left(\hat{\pi}_{t, T}^{w}-\hat{\delta}_{t, T}^{w}\right)\right\} .
$$


Quasi-differencing this expression yields

$$
\begin{aligned}
\hat{w}_{t}^{\star}-\hat{w}_{t}= & \frac{1-\beta \alpha_{w}}{1+\omega \theta_{w}}\left(\omega \hat{h}_{t}-\hat{\lambda}_{t}-\hat{w}_{t}\right)+\beta \alpha_{w} \mathrm{E}_{t}\left\{\hat{w}_{t+1}^{\star}-\hat{w}_{t+1}\right\} \\
& +\left(1-\beta \alpha_{w}\right)\left(\beta \alpha_{w}\right) \mathrm{E}_{t} \sum_{j=0}^{\infty}\left(\beta \alpha_{w}\right)^{j}\left\{\left(\hat{\pi}_{t, t+1+j}^{w}-\hat{\delta}_{t, t+1+j}^{w}\right)-\left(\hat{\pi}_{t+1, t+1+j}^{w}-\hat{\delta}_{t+1, t+1+j}^{w}\right)\right\}
\end{aligned}
$$

Now, notice that

$$
\hat{\delta}_{t+1, t+1+j}^{w}-\hat{\delta}_{t, t+1+j}^{w}=-\left[\left(1-\gamma_{w}\right) \hat{\pi}_{t+1}^{\star}+\gamma_{w} \hat{\pi}_{t}\right]
$$

and that

$$
\hat{\pi}_{t, t+1+j}^{w}-\hat{\pi}_{t+1, t+1+j}^{w}=\hat{\pi}_{t+1}^{w} .
$$

Thus, following the exact same steps as before

$$
\tilde{\pi}_{t}^{w}-\gamma_{w} \tilde{\pi}_{t-1}=\kappa_{w}\left(\omega \hat{h}_{t}-\hat{\lambda}_{t}-\hat{w}_{t}\right)+\beta \mathrm{E}_{t}\left\{\tilde{\pi}_{t+1}^{w}-\gamma_{w} \tilde{\pi}_{t}\right\}+\gamma_{w} \zeta_{t},
$$

where

$$
\tilde{\pi}_{t}^{w}=\hat{\pi}_{t}^{w}-\hat{\pi}_{t}^{\star}, \quad \tilde{\pi}_{t}=\hat{\pi}_{t}-\hat{\pi}_{t}^{\star}, \quad \zeta_{t}=\beta \mathrm{E}_{t}\left\{\Delta \hat{\pi}_{t+1}^{\star}\right\}-\Delta \hat{\pi}_{t}^{\star}, \quad \kappa_{w}=\frac{\left(1-\beta \alpha_{w}\right)\left(1-\alpha_{w}\right)}{\left(1+\omega \theta_{w}\right) \alpha_{w}} .
$$

Finally, wage inflation and inflation are linked together according to

$$
\tilde{\pi}_{t}^{w}=\tilde{\pi}_{t}+\hat{w}_{t}-\hat{w}_{t-1} .
$$

